Article

\title{
Phytochemistry and Comprehensive Chemical Profiling Study of Flavonoids and Phenolic Acids in the Aerial Parts of Allium Mongolicum Regel and Their Intestinal Motility Evaluation
}

\author{
Yongzhe Dong ${ }^{1,2,+}$, Jingya Ruan ${ }^{1,2,+}$, Zhijuan Ding ${ }^{2}$, Wei Zhao ${ }^{1}$, Mimi Hao ${ }^{2} \odot$, Ying Zhang ${ }^{2}$, \\ Hongyu Jiang ${ }^{2}$, Yi Zhang ${ }^{1,2, *}$ and Tao Wang ${ }^{1,2, *}$ \\ 1 Tianjin Key Laboratory of TCM Chemistry and Analysis, Tianjin University of Traditional Chinese Medicine, \\ 10 Poyanghu Road, West Area, Tuanbo New Town, Jinghai District, 301617 Tianjin, China; \\ dongyongzhe44@hotmail.com (Y.D.); Ruanjy19930919@163.com (J.R.); zhaowei126123@126.com (W.Z.) \\ 2 Institute of TCM, Tianjin University of Traditional Chinese Medicine, 10 Poyanghu Road, West Area, \\ Tuanbo New Town, Jinghai District, 301617 Tianjin, China; 15222792071@163.com (Z.D.); \\ haomimi126@126.com (M.H.); zyingtzy@163.com (Y.Z.); jhy15731602454@163.com (H.J.) \\ * Correspondence: zhwwxzh@tjutcm.edu.cn (Y.Z.); wangtao@tjutcm.edu.cn (T.W.); \\ Tel./Fax: +86-22-5959-6168 (T.W.) \\ + These authors contributed equally to this manuscript.
}

Academic Editor: Ping-Chung Kuo Received: 15 December 2019; Accepted: 24 January 2020; Published: 29 January 2020

\begin{abstract}
To clarify whether flavonoids and phenols in Allium mongolicum Regel have the effect of improving gastrointestinal function and analyze its quality, this study was designed to isolate and identify them from the aerial parts of A. mongolicum by using various chromatographic and spectrophotometric methods, a bioassay on motility of mouse isolated intestine tissue, as well as qualitative analysis using liquid chromatography/mass spectrometry (LC-MS) analysis. As a result, 31 flavonoids and phenolic acids were obtained and identified, including six new flavonoid glycosides, mongoflavonosides $A_{1}(1), A_{2}(2), A_{3}(3), A_{4}(4), B_{1}(5), B_{2}(6)$, and four new phenolic acid glycosides, mongophenosides $A_{1}(7), A_{2}(8), A_{3}(9), B(10)$. Among them, eleven flavonoids and three phenolic acids showed significant increase in the height of mouse small intestinal muscle. It was a first systematic bioactive constituents' study for A. mongolicum on gastrointestinal tract. Furthermore, according to the retention time $\left(t_{R}\right)$ and the exact mass-to-charge ratio $(\mathrm{m} / \mathrm{z})$, thirty-one compounds were unambiguously identified by comparing to the standard references by using LC-MS. Then, on the basis of generalized rules of MS/MS fragmentation pattern, chromatographic behaviors, as well as biosynthetic laws of the 31 isolates, five flavonoid glycosides and one phenolic acid glycoside were tentatively speculated. On the basis of the study, a fast analysis method for flavonoids and phenolic acids in A. mongolicum was established.
\end{abstract}

Keywords: Allium mongolicum Regel; flavonoids; phenolic acids; mouse isolated intestine tissue; qualitative analysis

\section{Introduction}

Flavonoids and phenolic acids are secondary metabolites found in most Allium vegetables, such as onion (Allium cepa L.) [1], scallion (Allium fistulosum L.) [2], garlic (Allium sativum L.) [3], fruits, and traditional medicine. They also exert multiple biological properties, such as antitumor $[4,5]$ antioxidant [6,7], anti-inflammation and gastrointestinal motility effect improvement [8-11], which make them show high correlations in the inhibition or management of many chronic diseases, 
such as cardiovascular and cerebrovascular diseases, diabetes, cancer, digestive system diseases, and so on.

As a traditional Mongolian medicinal herb, Allium mongolicum Regel (Liliaceae family) is mainly found in the high altitude desert of the Inner Mongolia Autonomous Region, Ningxia Hui Autonomous Region, Qinghai, Gansu, and Shanxi provinces [12], and has various special properties, such as stimulating the appetite, lowering blood pressure, hypolipidemic, replenishing the kidneys, and acting as an aphrodisiac [7]. Though it has been used to stimulate the appetite, studies were mainly focused on animal cultivation and improvement on meat products [12-14] up until now. Experiments related to the gastrointestinal tract have not been reported for the plant. On the other hand, pharmacological investigations showed the biological activities of A. mongolicum, including anti-inflammatory, antimicrobial, and antioxidant, which were attributed to the presence of different phytochemical groups like flavonoids and phenols $[7,15,16]$. However, it is still limited to the activity of total flavonoids and phenolic acids. Until now, the phytochemistry investigation of flavonoids is only reported by Dr. Zhao [16] and our laboratory [17,18] — there is no report for phenolic acids investigation. Moreover, there is no comprehensive chemical profiling study for the plant.

The present study was designed to isolate and identify flavonoids and phenolic acids from the aerial parts of $A$. mongolicum followed by bioactivity study on the motility of mouse isolated intestine tissue and qualitative analysis using liquid chromatography/mass spectrometry (LC-MS) analysis.

\section{Results and Discussion}

The fresh, aerial parts of $A$. mongolicum (17.8 kg) was successively heated under reflux with $95 \%$ EtOH for $3 \mathrm{~h}$ and $50 \% \mathrm{EtOH}$ for $2 \mathrm{~h}$ one time each to obtain dry extract of A. mongolicum aerial parts $(\mathrm{AM}, 515.0 \mathrm{~g})$. Then $470.1 \mathrm{~g}$ of it was partitioned with EtOAc/ $\mathrm{H}_{2} \mathrm{O}(1: 1,8 \mathrm{~L} / 8 \mathrm{~L})$ to yield EtOAc layer dry extract (AME, $64.9 \mathrm{~g}$ ) and $\mathrm{H}_{2} \mathrm{O}$ layer dry extract ( $\mathrm{AMH}, 381.0 \mathrm{~g}$ ).

Then, $\mathrm{AM}, \mathrm{AMH}$, and $\mathrm{AME}$ were tested for frequency and height by using a tissue perfusion method. As results, AM and AMH showed significant increase in the contraction amplitude of mouse small intestinal muscle at $200 \mu \mathrm{g} / \mathrm{mL}$ (relative height for AM: $137.4 \pm 11.8 \% *$ and AMH: $121.8 \pm 1.0 \% * *$, respectively), but had no significant effect on frequency (relative frequency for AM: $95.2 \pm 2.8 \%$ and AMH: $100.1 \pm 1.9 \%$, respectively). While AME displayed no significant effect on both of them (relative height: $127.9 \pm 20.8 \%$; relative frequency: $100.0 \pm 9.18 \%$ ).

Therefore, AMH was further fractionated by column chromatography (CC) and purified by HPLC to afford six new flavonoid glycosides, named as mongoflavonosides $A_{1}-A_{4}(\mathbf{1}-4)$, $B_{1}$ (5), $B_{2}$ (6), four new phenolic acid glycosides, named as mongophenosides $A_{1}-A_{3}$ (7-9), B (10) (Figure 1), along with known compounds, kaempferol-3,7,4'-tri-O- $\beta$-glucoside (18) [19], quercetin-3-O- $\beta$-D-rutinoside-7-O- $\beta$-D-glucuronide (25) [20], quercetin-3,7,4'-tri-O-glucoside (26) [21], isorhamnetin 3-O- $\beta$-D-glucopyranoside (27) [22], as well as three phenols, trans-p-hydroxycinnamate sophorose (28) [23], tuberonoid A (29) [24], trans-caffeic acid (30) [25], and benzyl-O- $\beta$-D-glucopyranoside (31) [26] (Figure 2). Among the known isolates, 28 and 30 were obtained from the Allium genus for the first time; 18, 25-27, 29, and 31 were firstly found from the plant.

Furthermore, the improved effects on the motility of the mouse isolated intestine tissue of the above-mentioned compounds as well as our previously reported flavonoids, kaempferol-3-O- $\beta$-D-glucopyranoside (11), kaempferol-3-O- $\beta$-D-glucopyranosyl $(1 \rightarrow 4)$ - $\beta$-D-glucopyranoside $(\mathbf{1 2})$, kaempferol-3-O- $\beta$-D- rutinoside (13), kaemperol-3-O- $\beta$-D-glucopyranosyl $(1 \rightarrow 4)[\alpha$-L-rhamanopyranosyl $(1 \rightarrow 6)]-\beta$-D-glucopyranoside (14), kaempferol-3-O-rutinoside-7-O-glucuronide (15), kaempferol-3-rutinoside-4'-glucopyranoside (16), kaempferol-3-O-gentiobioside- $4^{\prime}-O$-glucopyranoside

(17) quercetin-3-O-(6" -O-acetyl)- $\beta$-D-glucopyranoside quercetin-3-O- $\beta$-D-glucopyranosyl $(1 \rightarrow 4)-\beta$-D-glucopyranoside quercetin-3,4'-di-O- $\beta$-D-glucopyranoside
(23),
(21), rutin (20), (22), 
glucopyranoside-7-O- $\beta$-D-glucopyranoside (24) [18] (Figure 2) were reported here. Then, qualitative analysis for the aerial parts of A. mongolicum by using LC-MS spectrometry technology was developed.
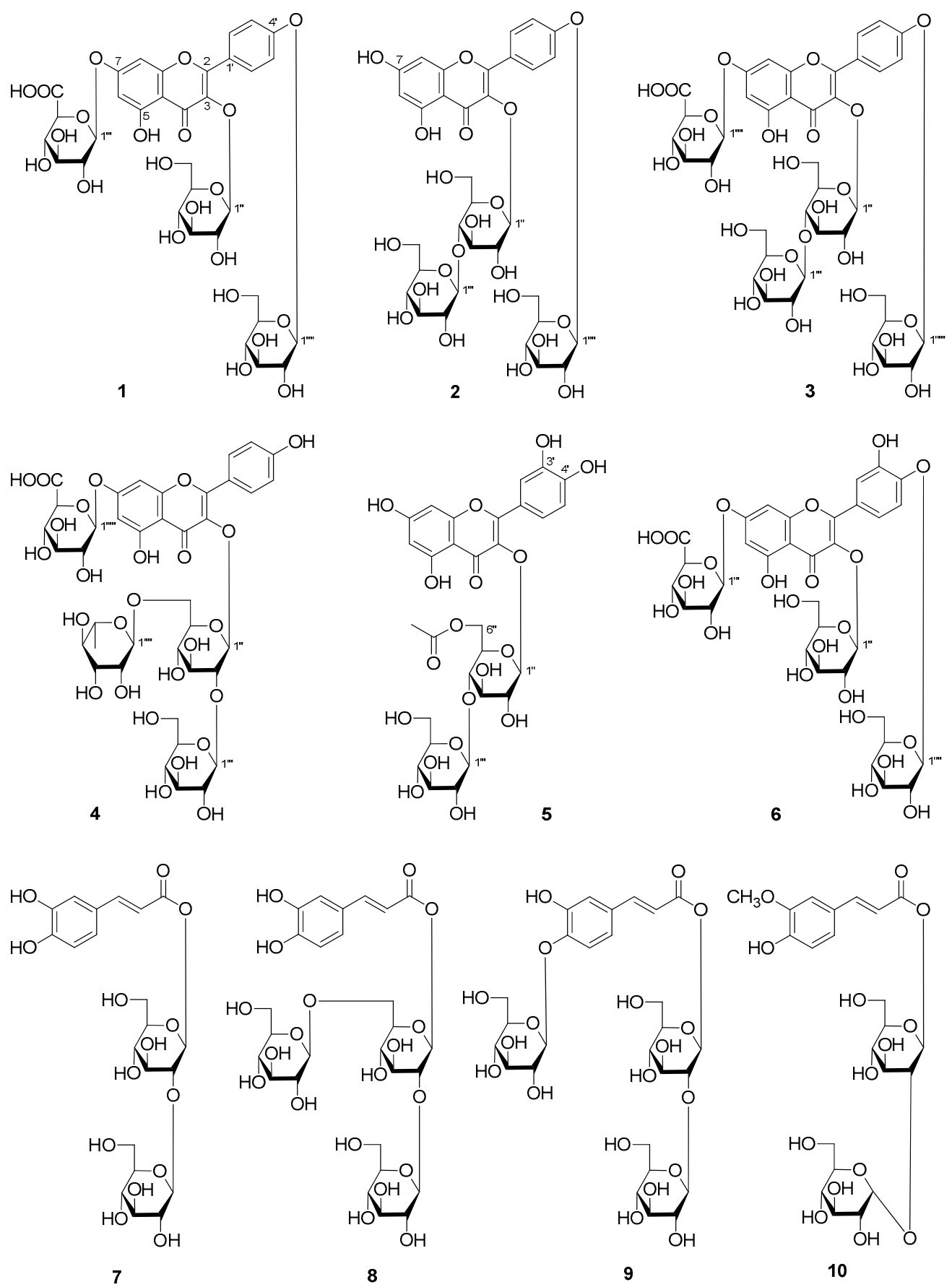

Figure 1. The new compounds obtained from the aerial parts of A. mongolicum (1-10). 

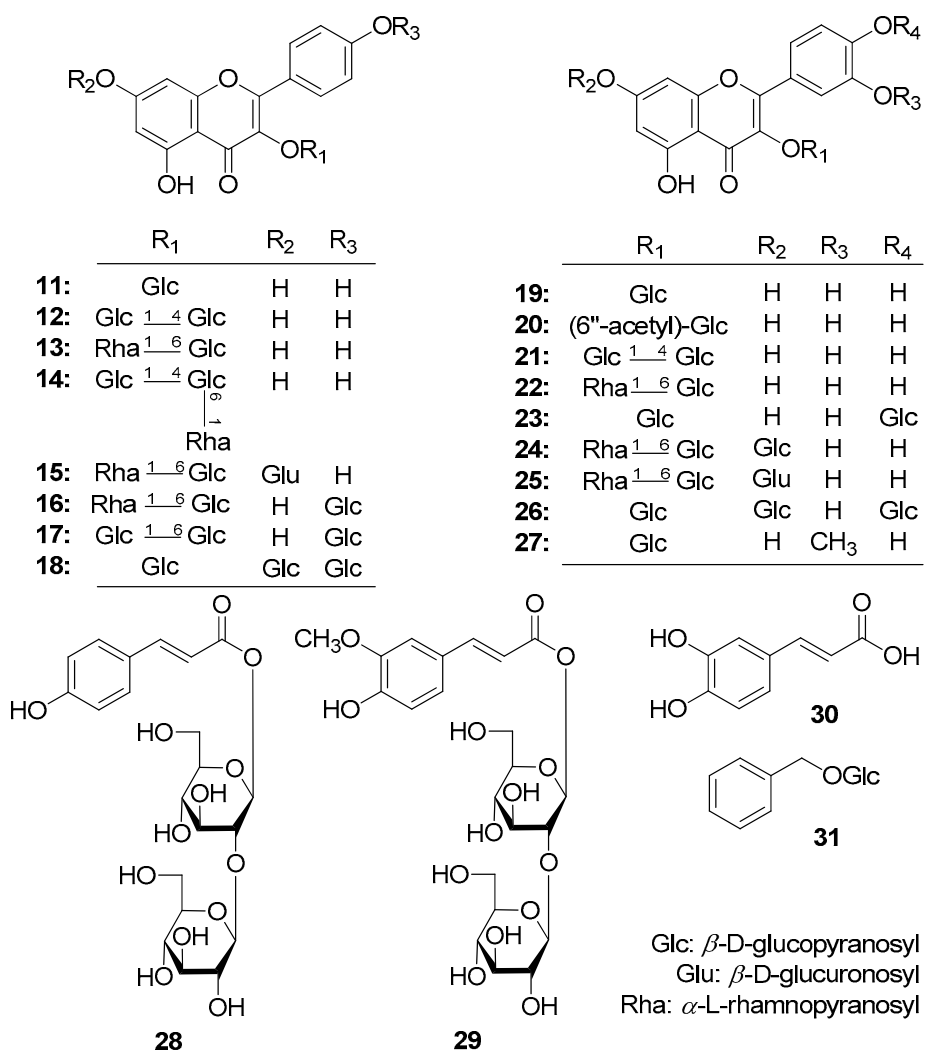

\begin{tabular}{llcccc}
$R_{3}$ & & $\mathrm{R}_{1}$ & $\mathrm{R}_{2}$ & $\mathrm{R}_{3}$ & $\mathrm{R}_{4}$ \\
\cline { 3 - 6 } $\mathrm{H}$ & 19: & Glc & $\mathrm{H}$ & $\mathrm{H}$ & $\mathrm{H}$ \\
$\mathrm{H}$ & 20: & 6"-acetyl)-Glc & $\mathrm{H}$ & $\mathrm{H}$ & $\mathrm{H}$ \\
$\mathrm{H}$ & 21: & Glc $\frac{1}{4} \mathrm{Glc}$ & $\mathrm{H}$ & $\mathrm{H}$ & $\mathrm{H}$ \\
$\mathrm{H}$ & 22: & Rha $\frac{16}{6} \mathrm{Glc}$ & $\mathrm{H}$ & $\mathrm{H}$ & $\mathrm{H}$ \\
& 23: & Glc & $\mathrm{H}$ & $\mathrm{H}$ & $\mathrm{Glc}$ \\
& 24: & Rha $\frac{16}{16} \mathrm{Glc}$ & $\mathrm{Glc}$ & $\mathrm{H}$ & $\mathrm{H}$ \\
$\mathrm{H}$ & 25: & Rha $\frac{16}{6} \mathrm{Glc}$ & $\mathrm{Glu}$ & $\mathrm{H}$ & $\mathrm{H}$ \\
$\mathrm{Glc}$ & 26: & Glc & $\mathrm{Glc}$ & $\mathrm{H}$ & $\mathrm{Glc}$ \\
$\mathrm{Glc}$ & 27: & Glc & $\mathrm{H}$ & $\mathrm{CH}_{3}$ & $\mathrm{H}$ \\
\hline
\end{tabular}

Figure 2. The known compounds obtained from the aerial parts of A. mongolicum (11-31).

\subsection{Identification of Compounds}

Mongoflavonoside $A_{1}(\mathbf{1})$ was isolated as a yellow powder with negative optical rotation $\left([\alpha]_{D}{ }^{25}-54.0, \mathrm{H}_{2} \mathrm{O}\right)$. Its molecular formula was deduced to be $\mathrm{C}_{33} \mathrm{H}_{38} \mathrm{O}_{22}$ by the negative-ion Electron Spray Ionization-Quadrupole-Orbitrap-Mass Spectrometry (ESI-Q-Orbitrap MS) analysis $(\mathrm{m} / \mathrm{z} 785.17883$ $[\mathrm{M}-\mathrm{H}]^{-}$, calculated for $\left.\mathrm{C}_{33} \mathrm{H}_{37} \mathrm{O}_{22}, 785.17710\right)$. The IR spectrum displayed the absorption bands assignable to hydroxyl $\left(3354 \mathrm{~cm}^{-1}\right)$, carbonyl $\left(1716 \mathrm{~cm}^{-1}\right), \alpha, \beta$-unsaturated ketone carbonyl $\left(1652 \mathrm{~cm}^{-1}\right)$, aromatic ring $\left(1601,1507,1457 \mathrm{~cm}^{-1}\right)$, and ether functions $\left(1072 \mathrm{~cm}^{-1}\right)$, respectively. The ${ }^{1} \mathrm{H}$ and ${ }^{13} \mathrm{C}-\mathrm{NMR}$ (Table 1) spectra suggested that 1 was a flavonoid glycoside with a kaempferol aglycone [ $6.48\left(1 \mathrm{H}\right.$, br. s, H-6), $6.88\left(1 \mathrm{H}\right.$, br. s, H-8), $7.19\left(2 \mathrm{H}, \mathrm{d}, J=9.0 \mathrm{~Hz}, \mathrm{H}-3^{\prime}, 5^{\prime}\right), 8.16(2 \mathrm{H}, \mathrm{d}, J=9.0 \mathrm{~Hz}$, $\left.\left.\mathrm{H}-2^{\prime}, 6^{\prime}\right)\right]$ and three glycosyl groups $\left[\delta 5.05\left(1 \mathrm{H}, \mathrm{d}, J=7.0 \mathrm{~Hz}, \mathrm{H}-1^{\prime \prime \prime \prime}\right), 5.26\left(1 \mathrm{H}, \mathrm{d}, J=6.5 \mathrm{~Hz}, \mathrm{H}-1^{\prime \prime \prime}\right)\right.$, $\left.5.50\left(1 \mathrm{H}, \mathrm{d}, J=7.0 \mathrm{~Hz}, \mathrm{H}-1^{\prime \prime}\right)\right]$. Acid hydrolysis of 1 with $5 \%$ aqueous $\mathrm{H}_{2} \mathrm{SO}_{4}$ solution-1,4-dioxane (1:1, $v / v)$ under $110{ }^{\circ} \mathrm{C}$ for $2 \mathrm{~h}$ to afford D-glucuronic acid and D-glucose, whose absolute configurations were determined by GC-MS analysis of their trimethysilyl thiazolidine derivatives [27]. Meanwhile, correlations were observed between the following proton and carbon pairs in its HSQC-TOCSY spectrum: $\mathrm{H}-1^{\prime \prime}$ and $\mathrm{C}-1^{\prime \prime}-\mathrm{C}-5^{\prime \prime} ; \delta_{\mathrm{H}} 3.35,3.58\left(\mathrm{H}_{2}-6^{\prime \prime}\right)$ and $\mathrm{C}-4^{\prime \prime}-\mathrm{C}-6^{\prime \prime} ; \mathrm{H}-1^{\prime \prime \prime}$ and $\mathrm{C}-1^{\prime \prime \prime}-\mathrm{C}-4^{\prime \prime \prime} ; \delta_{\mathrm{H}} 3.98$ $\left(\mathrm{H}-5^{\prime \prime \prime}\right)$ and $\mathrm{C}-2^{\prime \prime \prime}-\mathrm{C}-5^{\prime \prime \prime} ; \mathrm{H}-1^{\prime \prime \prime \prime}$ and $\mathrm{C}-2^{\prime \prime \prime \prime}-\mathrm{C}-4^{\prime \prime \prime \prime} ; \delta_{\mathrm{H}} 3.50\left(\mathrm{H}_{2}-6^{\prime \prime \prime \prime}\right)$ and $\mathrm{C}-4^{\prime \prime \prime \prime}-\mathrm{C}-6^{\prime \prime \prime \prime}$. Combining with the correlations displayed in its ${ }^{1} \mathrm{H}^{1} \mathrm{H}$ COSY and HSQC spectrum, the NMR data of three glycosyls were assigned in detail. Finally, according to the long-range correlations from $\mathrm{H}-1^{\prime \prime}$ to $\mathrm{C}-3 ; \mathrm{H}-1^{\prime \prime \prime}$ to $\mathrm{C}-7 ; \mathrm{H}-\mathrm{1}^{\prime \prime \prime \prime}$ to $\mathrm{C}-4^{\prime}$ showed in its HMBC experiment (Figure 3), the connections between glycosyl groups and aglycone were determined. On the basis of above-mentioned evidence, the structure of mongoflavonoside $A_{1}(\mathbf{1})$ was identified. 
Table 1. ${ }^{13} \mathrm{C}-\mathrm{NMR}$ data for $1-6$ in DMSO- $d_{6}$.

\begin{tabular}{cccccccccccccc}
\hline No. & $\mathbf{1}$ & $\mathbf{2}$ & $\mathbf{3}$ & $\mathbf{4}$ & $\mathbf{5}$ & $\mathbf{6}$ & $\mathbf{N o .}$ & $\mathbf{1}$ & $\mathbf{2}$ & $\mathbf{3}$ & $\mathbf{4}$ & $\mathbf{5}$ & $\mathbf{6}$ \\
\hline 2 & 156.0 & 155.5 & 156.1 & 157.9 & 156.3 & 156.0 & COCH $_{3}$ & & & & & 169.6 \\
3 & 133.9 & 133.5 & 133.9 & 133.1 & 132.8 & 134.0 & COCH $_{3}$ & & & & 19.9 & \\
4 & 177.7 & 177.3 & 177.6 & 177.3 & 177.0 & 177.7 & $1^{\prime \prime \prime}$ & 99.0 & 103.0 & 103.0 & 103.4 & 103.2 & 99.2 \\
5 & 160.8 & 161.1 & 160.7 & 160.4 & 161.1 & 160.7 & $2^{\prime \prime \prime}$ & 72.6 & 73.2 & 73.1 & 73.0 & 73.0 & 72.7 \\
6 & 99.2 & 98.9 & 99.4 & 99.3 & 98.8 & 99.2 & $3^{\prime \prime \prime}$ & 75.7 & 76.3 & 76.3 & 76.3 & 76.3 & 76.1 \\
7 & 162.5 & 164.8 & 162.9 & 162.3 & 164.9 & 162.7 & $4^{\prime \prime \prime}$ & 71.3 & 69.9 & 69.9 & 69.9 & 69.9 & 71.6 \\
8 & 94.3 & 93.8 & 94.4 & 94.6 & 93.6 & 94.3 & $5^{\prime \prime \prime}$ & 75.0 & 76.7 & 76.7 & 76.7 & 76.7 & 74.3 \\
9 & 156.2 & 156.4 & 156.0 & 156.2 & 156.5 & 156.0 & $6^{\prime \prime \prime}$ & 170.5 & 60.9 & 60.9 & 60.9 & 61.0 & 171.4 \\
10 & 105.8 & 103.8 & 105.6 & 105.6 & 103.4 & 105.6 & $1^{\prime \prime \prime \prime}$ & 99.8 & 99.9 & 99.3 & 101.0 & 101.3 \\
$1^{\prime}$ & 123.5 & 123.6 & 123.4 & 120.7 & 120.8 & 124.2 & $2^{\prime \prime \prime \prime}$ & 73.1 & 73.1 & 72.8 & 70.4 & 73.2 \\
$2^{\prime}$ & 130.6 & 130.5 & 130.6 & 130.9 & 116.0 & 116.5 & $3^{\prime \prime \prime \prime}$ & 76.4 & 76.4 & 76.2 & 70.9 & \\
$3^{\prime}$ & 115.7 & 115.7 & 115.7 & 115.0 & 144.8 & 146.0 & $4^{\prime \prime \prime \prime}$ & 69.5 & 69.5 & 71.7 & 71.6 & 75.7 \\
$4^{\prime}$ & 159.2 & 159.1 & 159.3 & 159.9 & 148.5 & 147.5 & $5^{\prime \prime \prime \prime \prime}$ & 77.0 & 77.0 & 73.9 & 68.1 & 69.6 \\
$5^{\prime}$ & 115.7 & 115.7 & 115.7 & 115.0 & 115.0 & 115.3 & $6^{\prime \prime \prime \prime \prime}$ & 60.5 & 60.5 & 171.4 & 17.5 & & 67.1 \\
$6^{\prime}$ & 130.6 & 130.5 & 130.6 & 130.9 & 121.3 & 121.0 & $1^{\prime \prime \prime \prime \prime}$ & & & 99.8 & 98.7 & & 60.6 \\
$1^{\prime \prime}$ & 100.6 & 100.5 & 100.4 & 100.8 & 100.5 & 100.5 & $2^{\prime \prime \prime \prime \prime}$ & & & 73.1 & 72.8 & & \\
$2^{\prime \prime}$ & 74.1 & 73.8 & 73.1 & 73.7 & 73.5 & 74.0 & $3^{\prime \prime \prime \prime \prime}$ & & & 76.4 & 76.2 & & \\
$3^{\prime \prime}$ & 76.3 & 74.6 & 74.6 & 74.6 & 74.5 & 76.4 & $4^{\prime \prime \prime \prime \prime}$ & & & 69.5 & 71.6 & & \\
$4^{\prime \prime}$ & 69.8 & 80.0 & 80.0 & 80.8 & 80.0 & 69.9 & $5^{\prime \prime \prime \prime \prime}$ & & & 77.0 & 73.7 & & \\
$5^{\prime \prime}$ & 77.5 & 75.3 & 75.4 & 73.7 & 71.9 & 77.6 & $6^{\prime \prime \prime \prime \prime}$ & & & 60.5 & 171.7 & & \\
$6^{\prime \prime}$ & 60.8 & 60.1 & 60.0 & 67.0 & 62.1 & 60.8 & & & & & & \\
\hline
\end{tabular}
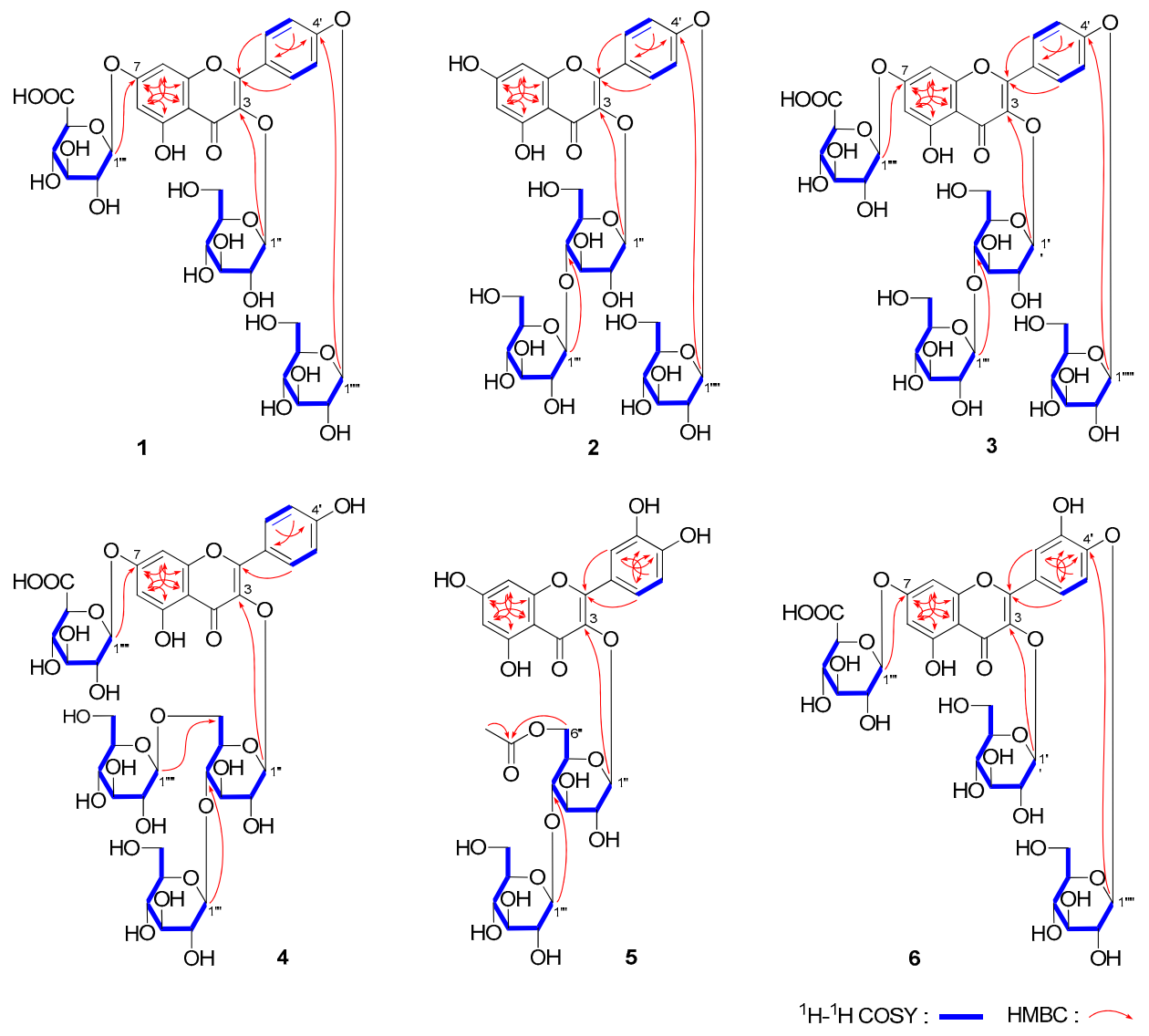

Figure 3. Key ${ }^{1} \mathrm{H}^{1} \mathrm{H}$ COSY and $\mathrm{HMBC}$ correlations of 1-6.

Mongoflavonoside $\mathrm{A}_{2}(2)$ is a yellow powder with negative optical rotation $\left([\alpha]_{\mathrm{D}}{ }^{25}-26.0, \mathrm{MeOH}\right)$. Its ESI-Q-Orbitrap MS spectrum showed a peak at $m / z 771.19971[\mathrm{M}-\mathrm{H}]^{-}$(calculated for $\mathrm{C}_{33} \mathrm{H}_{39} \mathrm{O}_{21}$, 771.19783), and its molecular formula was deduced to be $\mathrm{C}_{33} \mathrm{H}_{40} \mathrm{O}_{21}$. After hydrolyzing with $1 \mathrm{M} \mathrm{HCl}$, 
the product was analyzed by using HPLC with an optical rotation detector. As a result, D-glucose was detected [28]. The ${ }^{1} \mathrm{H}$ and ${ }^{13} \mathrm{C}-\mathrm{NMR}$ (Table 1) spectra suggested that compound 2 had the same aglycone, kaempferol [ $\delta 6.21$ (1H, br. s, H-6), 6.44 (1H, br. s, H-8), 7.17 (2H, d, J = 9.0 Hz, H-3' , 5' $), 8.11$ $\left.\left(2 \mathrm{H}, \mathrm{d}, J=9.0 \mathrm{~Hz}, \mathrm{H}-2^{\prime}, 6^{\prime}\right)\right]$ as that of 1 . In addition, there were three $\beta$-D-glucopyranosyl moieties [ $\left.\delta 4.27\left(1 \mathrm{H}, \mathrm{d}, J=8.0 \mathrm{~Hz}, \mathrm{H}-1^{\prime \prime \prime}\right), 5.03\left(1 \mathrm{H}, \mathrm{d}, J=7.5 \mathrm{~Hz}, \mathrm{H}-1^{\prime \prime \prime \prime \prime}\right), 5.51\left(1 \mathrm{H}, \mathrm{d}, J=8.0 \mathrm{~Hz}, \mathrm{H}-1^{\prime \prime}\right)\right]$. To solve the overlapping problem of three $\beta$-D-glucopyranosyl groups, the HSQC-TOCSY experiment was performed. The correlations between $\mathrm{C}-1^{\prime \prime}$ and $\delta_{\mathrm{H}} 3.26\left(\mathrm{H}-2^{\prime \prime}\right), 3.39\left(\mathrm{H}-4^{\prime \prime}\right), 3.41\left(\mathrm{H}-3^{\prime \prime}\right), 5.51$ $\left(\mathrm{H}-1^{\prime \prime}\right) ; \delta_{\mathrm{H}} 3.51,3.63\left(\mathrm{H}_{2}-6^{\prime \prime}\right)$ and $\mathrm{C}-4^{\prime \prime}-\mathrm{C}-6^{\prime \prime} ; \mathrm{H}-1^{\prime \prime \prime}$ and $\mathrm{C}-1^{\prime \prime \prime}-\mathrm{C}-4^{\prime \prime \prime} ; \delta_{\mathrm{H}} 3.42,3.71\left(\mathrm{H}_{2}-6^{\prime \prime \prime}\right)$ and $\mathrm{C}-4^{\prime \prime \prime}-\mathrm{C}-6^{\prime \prime \prime} ; \mathrm{H}-1^{\prime \prime \prime \prime \prime}$ and $\mathrm{C}-1^{\prime \prime \prime \prime \prime}-\mathrm{C}-4^{\prime \prime \prime \prime \prime} ; \delta_{\mathrm{H}} 3.50,3.71\left(\mathrm{H}_{2}-6^{\prime \prime \prime \prime \prime}\right)$ and $\mathrm{C}-4^{\prime \prime \prime \prime \prime}-\mathrm{C}-6^{\prime \prime \prime \prime \prime}$ were found in it. Moreover, the HMBC displayed the long-range correlations from $\mathrm{H}-1^{\prime \prime}$ to $\mathrm{C}-3$; $\mathrm{H}-1^{\prime \prime \prime}$ to $\mathrm{C}-4^{\prime \prime}$; $\mathrm{H}-1^{\prime \prime \prime \prime \prime}$ to $\mathrm{C}-4^{\prime}$ (Figure 3). Consequently, the structure of mongoflavonoside $\mathrm{A}_{2}(2)$ was elucidated to be kaempferol 3-O- $\beta$-D-glucopyranosyl $(1 \rightarrow 4)-\beta$-D-glucopyranosyl-4'-O- $\beta$-D-glucopyranoside.

Mongoflavonoside $\mathrm{A}_{3}$ (3) exhibited negative optical rotation $\left([\alpha]_{\mathrm{D}}{ }^{25}-64.7, \mathrm{H}_{2} \mathrm{O}\right)$. Its molecular formula was revealed to be $\mathrm{C}_{39} \mathrm{H}_{48} \mathrm{O}_{27}$ by negative ESI-Q-Orbitrap MS analysis $(\mathrm{m} / z$ 947.23242 [M $\mathrm{H}]^{-}$, calculated for $\left.\mathrm{C}_{39} \mathrm{H}_{47} \mathrm{O}_{27}, 947.22992\right)$. The ${ }^{1} \mathrm{H},{ }^{13} \mathrm{C}-\mathrm{NMR}$ (Table 1) along with various $2 \mathrm{D}$ NMR $\left({ }^{1} \mathrm{H}\right.$ ${ }^{1} \mathrm{H}$ COSY, HSQC, HMBC, and HSQC-TOCSY) spectra denoted that 3 had the same moiety, kaempferol 3-O- $\beta$-D-glucopyranosyl $(1 \rightarrow 4)$ - $\beta$-D-glucopyranosyl-4'-O- $\beta$-D-glucopyranosyl [ $\delta 4.26(1 \mathrm{H}, \mathrm{d}, J=7.0 \mathrm{~Hz}$, H-1'”'), $5.04\left(1 \mathrm{H}, \mathrm{d}, J=7.0 \mathrm{~Hz}, \mathrm{H}-1^{\prime \prime \prime \prime \prime}\right), 5.53\left(1 \mathrm{H}, \mathrm{d}, J=7.5 \mathrm{~Hz}, \mathrm{H}-1^{\prime \prime}\right), 6.46(1 \mathrm{H}$, br. s, H-6), $6.85(1 \mathrm{H}$, br. s, H-8), $\left.7.18\left(2 \mathrm{H}, \mathrm{d}, J=8.5 \mathrm{~Hz}, \mathrm{H}-3^{\prime}, 5^{\prime}\right), 8.14\left(2 \mathrm{H}, \mathrm{d}, J=8.5 \mathrm{~Hz}, \mathrm{H}-2^{\prime}, 6^{\prime}\right)\right]$ as that of 2. Meanwhile, one more $\beta$-D-glucuropyranosyl $\left[\delta 5.12\left(1 \mathrm{H}, \mathrm{d}, J=7.0 \mathrm{~Hz}, \mathrm{H}-1^{\prime \prime \prime \prime}\right)\right]$ appeared in 3 . On the other hand, the proton signals at H-6 and H-8 and the carbon signal at C-7 shifted to the lower field in comparison with those of 2 , which suggested that the $\beta$-D-glucuropyranosyl linked with 7-position of kaempferol. It was clarified by the long-range correlation from $\mathrm{H}-1^{\prime \prime \prime \prime}$ to $\mathrm{C}-7$ (Figure 3). Then, the structure of mongoflavonoside $\mathrm{A}_{3}$ (3) was determined.

The molecular formula of mongoflavonoside $\mathrm{A}_{4}(4)$ was measured to be $\mathrm{C}_{39} \mathrm{H}_{48} \mathrm{O}_{26}$ by negative ESI-Q-Orbitrap MS analysis $(m / z \text { 931.23785 [M - H] }]^{-}$, (calculated for $\left.\mathrm{C}_{39} \mathrm{H}_{47} \mathrm{O}_{26}, 931.23501\right)$. Its acid hydrolysis product was derived to obtain trimethylsilane thiazolidine derivatives, then the existence of D-glucuronic acid, D-glucose, and L-rhamnose were clarified by GC analysis [27]. Its ${ }^{1} \mathrm{H}$, ${ }^{13}$ C-NMR (Table 1) and 2D NMR spectra indicated that 4 had the same moiety, kaempferol 3-O- $\beta$-D-glucopyranosyl $(1 \rightarrow 4)[\alpha$-L-rhamanopyranosyl $(1 \rightarrow 6)]-\beta$-D-glucopyranosyl $[\delta \quad 4.14(1 \mathrm{H}, \mathrm{d}$,

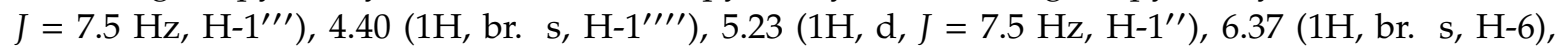
$6.73\left(1 \mathrm{H}\right.$, br. s, H-8), $\left.6.88\left(2 \mathrm{H}, \mathrm{d}, J=9.0 \mathrm{~Hz}, \mathrm{H}-3^{\prime}, 5^{\prime}\right), 8.00\left(2 \mathrm{H}, \mathrm{d}, J=9.0 \mathrm{~Hz}, \mathrm{H}-2^{\prime}, 6^{\prime}\right)\right]$ as that of kaempferol 3-O- $\beta$-D-glucopyranosyl $(1 \rightarrow 4)$ [ $\alpha$-L-rhamanopyranosyl $(1 \rightarrow 6)]$ - $\beta$-D-glucopyranoside [29]. In addition, one $\beta$-D-glucuropyranosyl $\left[\delta_{\mathrm{H}} 5.16\left(1 \mathrm{H}, \mathrm{d}, J=7.0 \mathrm{~Hz}, \mathrm{H}-1^{\prime \prime \prime \prime \prime}\right) ; \delta_{\mathrm{C}} 71.6\right.$ $\left.\left(\mathrm{C}-4^{\prime \prime \prime \prime \prime}\right), 72.8\left(\mathrm{C}-2^{\prime \prime \prime \prime \prime}\right), 73.7\left(\mathrm{C}-5^{\prime \prime \prime \prime \prime}\right), 76.2\left(\mathrm{C}-3^{\prime \prime \prime \prime \prime}\right), 98.7\left(\mathrm{C}-1^{\prime \prime \prime \prime \prime}\right), 171.7\left(\mathrm{C}-6^{\prime \prime \prime \prime \prime}\right)\right]$ appeared in 4. The long-range correlation observation from $\mathrm{H}-1^{\prime \prime \prime \prime \prime}$ to $\mathrm{C}-7$ (Figure 3 ) in its HMBC spectrum suggested the $\beta$-D-glucuropyranosyl connected with C-7 of kaempferol 3-O- $\beta$-D-glucopyranosyl $(1 \rightarrow 4)[\alpha$-L-rhamanopyranosyl $(1 \rightarrow 6)]-\beta$-D-glucopyranosyl. Then, the structure of mongoflavonoside $\mathrm{A}_{4}(4)$ was constructed.

Mongoflavonoside $\mathrm{B}_{1}(5)$ was isolated as a yellow powder and showed negative optical rotation $\left([\alpha]_{\mathrm{D}}{ }^{25}-12.0, \mathrm{MeOH}\right)$. The molecular formula, $\mathrm{C}_{29} \mathrm{H}_{32} \mathrm{O}_{18}$ of 5 was determined from ESI-Q-Orbitrap MS (m/z 667.15228 [M - H] $]^{-}$; calculated for $\left.\mathrm{C}_{29} \mathrm{H}_{31} \mathrm{O}_{18}, 667.15049\right)$ analysis. Its IR spectrum exhibited characteristic absorptions of hydroxyl $\left(3362 \mathrm{~cm}^{-1}\right)$, ester carbonyl $\left(1721 \mathrm{~cm}^{-1}\right), \alpha, \beta$-unsaturated ketone carbonyl $\left(1654 \mathrm{~cm}^{-1}\right)$, aromatic ring $\left(1605,1507,1448 \mathrm{~cm}^{-1}\right)$, and ether functions $\left(1070 \mathrm{~cm}^{-1}\right)$. The ${ }^{1} \mathrm{H}$ and ${ }^{13} \mathrm{C}-\mathrm{NMR}$ spectra displayed signals of a quercetin moiety $[\delta 6.18(1 \mathrm{H}, \mathrm{br} . \mathrm{s}, \mathrm{H}-6), 6.39(1 \mathrm{H}, \mathrm{br}$. s, H-8), $\left.6.83\left(1 \mathrm{H}, \mathrm{d}, J=8.5 \mathrm{~Hz}, \mathrm{H}-5^{\prime}\right), 7.50\left(1 \mathrm{H}, \mathrm{dd}, J=2.0,8.5 \mathrm{~Hz}, \mathrm{H}-6^{\prime}\right), 7.51\left(1 \mathrm{H}, \mathrm{d}, J=2.0 \mathrm{~Hz}, \mathrm{H}-2^{\prime}\right)\right]$, two $\beta$-D-glucopyranosyl groups [ $\left.\delta 4.21\left(1 \mathrm{H}, \mathrm{d}, J=8.0 \mathrm{~Hz}, \mathrm{H}-1^{\prime \prime \prime}\right), 5.40\left(1 \mathrm{H}, \mathrm{d}, J=8.0 \mathrm{~Hz}, \mathrm{H}-1^{\prime \prime}\right)\right]$, along with an acetyl $\left[\delta_{\mathrm{H}} 1.71\left(3 \mathrm{H}, \mathrm{s}, 6^{\prime \prime}-\mathrm{COCH}_{3}\right) ; \delta_{\mathrm{C}} 19.9\left(6^{\prime \prime}-\mathrm{COCH}_{3}\right), 169.6\left(6^{\prime \prime}-\mathrm{COCH}_{3}\right)\right]$. As shown in Figure 3 , the ${ }^{1} \mathrm{H}^{1} \mathrm{H}$ COSY experiment on 5 indicated the presence of partial structures written in bold lines. Moreover, in the HMBC spectrum, the long-range correlations from $\mathrm{H}-1^{\prime \prime}$ to $\mathrm{C}-3$; $\mathrm{H}-1^{\prime \prime \prime}$ to $\mathrm{C}-4^{\prime \prime}$; 
$6^{\prime \prime}-\mathrm{COCH}_{3}$ to $6^{\prime \prime}-\mathrm{COCH}_{3} ; \mathrm{H}_{2}-6^{\prime \prime}$ to $6^{\prime \prime}-\mathrm{COCH}_{3}$ were observed. Finally, after treating 5 with $1 \mathrm{M} \mathrm{HCl}$, D-glucose was detected from its acid hydrolysis product [28]. Consequently, the structure of 5 was identified, and named as mongoflavonoside $\mathrm{B}_{1}$.

The molecular formula, $\mathrm{C}_{33} \mathrm{H}_{38} \mathrm{O}_{23}$ of 6 was measured on ESI-Q-Orbitrap MS ( $\mathrm{m} / \mathrm{z} 801.17407$ [M $-\mathrm{H}]^{-}$, calculated for $\mathrm{C}_{33} \mathrm{H}_{37} \mathrm{O}_{23}, 801.17201$ ) analysis. The ${ }^{1} \mathrm{H},{ }^{13} \mathrm{C}$ NMR (Table 1 ) and 2D NMR $\left({ }^{1} \mathrm{H}\right.$ ${ }^{1} \mathrm{H}$ COSY, HSQC, HMBC, HSQC-TOCSY) spectra suggested $\mathbf{6}$ had the same glycosyl moieties with 1: two $\beta$-D-glucopyranosyls [ $\left.\delta 4.88\left(1 \mathrm{H}, \mathrm{d}, J=7.0 \mathrm{~Hz}, \mathrm{H}-1^{\prime \prime \prime \prime}\right), 5.52\left(1 \mathrm{H}, \mathrm{d}, J=7.0 \mathrm{~Hz}, \mathrm{H}-1^{\prime \prime}\right)\right]$, and one $\beta$-D-glucuropyranosyl [5.16 $\left.\left(1 \mathrm{H}, \mathrm{d}, J=7.0 \mathrm{~Hz}, \mathrm{H}-\mathrm{1}^{\prime \prime \prime}\right)\right]$. Meanwhile, 6 possessed the same aglycone, quercetin $\left[\delta 6.45\left(1 \mathrm{H}\right.\right.$, br. s, H-6), $6.85\left(1 \mathrm{H}\right.$, br. s, H-8), $7.23\left(1 \mathrm{H}, \mathrm{d}, J=8.5 \mathrm{~Hz}, \mathrm{H}-5^{\prime}\right), 7.64(1 \mathrm{H}, \mathrm{d}, J=1.5$, $\left.\left.8.5 \mathrm{~Hz}, \mathrm{H}-6^{\prime}\right), 7.69\left(1 \mathrm{H}, \mathrm{d}, J=1.5 \mathrm{~Hz}, \mathrm{H}-2^{\prime}\right)\right]$ as that of 5 . Finally, the connectivities of glycosyl moieties with aglycone were determined by the correlations from $\mathrm{H}-1^{\prime \prime}$ to $\mathrm{C}-3 ; \mathrm{H}-1^{\prime \prime \prime}$ to $\mathrm{C}-7 ; \mathrm{H}-1^{\prime \prime \prime \prime}$ to $\mathrm{C}-4^{\prime}$ (Figure 3) showed in its HMBC spectrum.

Mongophenoside $A_{1}(7)$ was obtained as a white powder with negative optical rotation $\left([\alpha]_{D}{ }^{25}\right.$ $-21.0, \mathrm{MeOH})$. ESI-Q-Orbitrap MS of 7 exhibited quasimolecular ion peak at $\mathrm{m} / z 503.14151[\mathrm{M}-\mathrm{H}]^{-}$ (calculated for $\mathrm{C}_{21} \mathrm{H}_{27} \mathrm{O}_{14}, 503.13953$ ), and its molecular formula was deduced to be $\mathrm{C}_{21} \mathrm{H}_{28} \mathrm{O}_{14}$. The IR spectrum of it showed absorption bands ascribable to hydroxyl $\left(3362 \mathrm{~cm}^{-1}\right), \alpha, \beta$-unsaturated ester carbonyl $\left(1709 \mathrm{~cm}^{-1}\right)$, aromatic ring $\left(1601,1521,1447 \mathrm{~cm}^{-1}\right)$, and ether function $\left(1074 \mathrm{~cm}^{-1}\right)$. Acid hydrolysis of 7 liberated D-glucose, which was identified by HPLC analysis [28]. Its ${ }^{1} \mathrm{H},{ }^{13} \mathrm{C}$ NMR (Table 2) spectra indicated the existence of one trans-caffeoyl $\left[\delta_{\mathrm{H}} 6.27(1 \mathrm{H}, \mathrm{d}, J=16.0 \mathrm{~Hz}, \mathrm{H}-8), 6.75(1 \mathrm{H}\right.$, d, $J=7.5 \mathrm{~Hz}, \mathrm{H}-5), 7.01(1 \mathrm{H}$, br. d, ca. $J=8 \mathrm{~Hz}, \mathrm{H}-6), 7.06(1 \mathrm{H}$, br. s, H-2), $7.55(1 \mathrm{H}, \mathrm{d}, J=16.0 \mathrm{~Hz}$, $\mathrm{H}-7) ; \delta_{\mathrm{C}} 113.3$ (C-8), $146.2(\mathrm{C}-7), 164.9$ (C-9)], along with two $\beta$-D-glucopyranosyl groups [ $\delta 4.42(1 \mathrm{H}, \mathrm{d}$, $\left.\left.J=8.0 \mathrm{~Hz}, \mathrm{H}-1^{\prime \prime}\right), 5.56\left(1 \mathrm{H}, \mathrm{d}, J=8.0 \mathrm{~Hz}, \mathrm{H}-1^{\prime}\right)\right]$. Meanwhile, the partial structures written in bold lines shown in Figure 4 were determined by proton and proton correlations observed in its ${ }^{1} \mathrm{H}^{1} \mathrm{H}$ COSY experiment. The planar structure of 5 was finally elucidated according to the long-range correlations from $\mathrm{H}-1^{\prime}$ to $\mathrm{C}-9$; $\mathrm{H}-1^{\prime \prime}$ to $\mathrm{C}-2^{\prime}$ (Figure 4) found in HMBC experiment, and the structure of 7 was named as mongophenoside $\mathrm{A}_{1}$.

Table 2. ${ }^{13} \mathrm{C}-\mathrm{NMR}$ data for $\mathbf{7 - 1 0}$.

\begin{tabular}{cccccccccc}
\hline No. & $\mathbf{7}^{\boldsymbol{a}}$ & $\mathbf{8}^{\boldsymbol{a}}$ & $\mathbf{9}^{\boldsymbol{a}}$ & $\mathbf{1 0}^{\boldsymbol{b}}$ & $\mathbf{N o .}$ & $\mathbf{7}^{\boldsymbol{a}}$ & $\mathbf{8}^{\boldsymbol{a}}$ & $\mathbf{9}^{\boldsymbol{a}}$ & $\mathbf{1 0}^{\boldsymbol{b}}$ \\
\hline 1 & 125.4 & 125.2 & 128.5 & 127.5 & $5^{\prime}$ & 77.4 & 75.9 & 77.1 & 78.0 \\
2 & 114.6 & 114.6 & 115.0 & 111.7 & $6^{\prime}$ & 60.3 & 67.7 & 60.6 & 62.8 \\
3 & 145.5 & 145.6 & 146.7 & 149.5 & $1^{\prime \prime}$ & 104.4 & 104.4 & 92.4 & 94.0 \\
4 & 148.6 & 149.0 & 147.5 & 151.0 & $2^{\prime \prime}$ & 74.4 & 74.4 & 81.6 & 73.9 \\
5 & 115.6 & 115.6 & 115.9 & 116.6 & $3^{\prime \prime}$ & 76.0 & 76.1 & 75.6 & 74.9 \\
6 & 121.6 & 121.7 & 120.9 & 124.2 & $4^{\prime \prime}$ & 69.3 & 69.3 & 69.0 & 71.8 \\
7 & 146.2 & 146.3 & 145.5 & 146.9 & $5^{\prime \prime}$ & 76.6 & 76.7 & 77.5 & 73.0 \\
8 & 113.3 & 113.1 & 115.5 & 115.1 & $6^{\prime \prime}$ & 60.3 & 60.3 & 60.3 & 62.9 \\
9 & 164.9 & 164.9 & 164.7 & 169.8 & $1^{\prime \prime \prime}$ & & 103.0 & 104.5 & \\
$3-\mathrm{OCH} 3$ & & & & 56.5 & $2^{\prime \prime \prime}$ & & 73.4 & 74.5 & \\
$1^{\prime \prime}$ & 92.3 & 92.3 & 101.4 & 98.2 & $3^{\prime \prime \prime}$ & & 76.6 & 76.0 & \\
$2^{\prime}$ & 81.5 & 81.4 & 73.1 & 76.3 & $4^{\prime \prime \prime}$ & & 69.9 & 69.3 & \\
$3^{\prime}$ & 75.6 & 75.5 & 75.7 & 78.1 & $5^{\prime \prime \prime}$ & & 76.8 & 76.6 & \\
$4^{\prime \prime}$ & 69.0 & 68.8 & 69.7 & 71.9 & $6^{\prime \prime \prime}$ & & 60.9 & 60.3 \\
\hline
\end{tabular}

Determined in ${ }^{a} \mathrm{DMSO}-d_{6},{ }^{b} \mathrm{CD}_{3} \mathrm{OD}$.

Mongophenoside $A_{2}(8)$, a white powder, showed negative optical rotation $\left([\alpha]_{D}^{25}-14.5, \mathrm{MeOH}\right)$. ESI-Q-Orbitrap MS analysis suggested its molecular formula was $\mathrm{C}_{27} \mathrm{H}_{38} \mathrm{O}_{19}\left(665.19427\left[\mathrm{M}-\mathrm{H}^{-}\right.\right.$; calculated for $\mathrm{C}_{27} \mathrm{H}_{37} \mathrm{O}_{19}, 665.19236$ ). The ${ }^{1} \mathrm{H}$ and ${ }^{13} \mathrm{C}-\mathrm{NMR}$ (Table 2) spectra indicated 8 possessed the same moiety, trans-caffeic acid-9-O- $\beta$-D-glucopyranosyl $(1 \rightarrow 2)-\beta$-D-glucopyranosyl $[\delta 4.42(1 \mathrm{H}, \mathrm{d}$, $\left.J=7.5 \mathrm{~Hz}, \mathrm{H}-1^{\prime \prime}\right), 5.56\left(1 \mathrm{H}, \mathrm{d}, J=7.0 \mathrm{~Hz}, \mathrm{H}-1^{\prime}\right), 6.27(1 \mathrm{H}, \mathrm{d}, J=16.0 \mathrm{~Hz}, \mathrm{H}-8), 6.76(1 \mathrm{H}, \mathrm{d}, J=7.5 \mathrm{~Hz}$, $\mathrm{H}-5), 7.02(1 \mathrm{H}$, br. d, ca. $J=8 \mathrm{~Hz}, \mathrm{H}-6), 7.06(1 \mathrm{H}$, br. s, H-2), $7.55(1 \mathrm{H}, \mathrm{d}, J=16.0 \mathrm{~Hz}, \mathrm{H}-7)]$ as that of 7. Except for that, one more $\beta$-D-glucopyranosyl [ $\left.\delta 4.17\left(1 \mathrm{H}, \mathrm{d}, J=7.5 \mathrm{~Hz}, \mathrm{H}-1^{\prime \prime \prime}\right)\right]$ appeared 
in 8. Meanwhile, $C-6^{\prime}$ of it was found to significantly shift to lower field ( $\delta_{C} 67.7$ for $8 ; 60.3$ for 7 ) comparing with 7 , which suggested C- $6^{\prime}$ was substituted by the $\beta$-D-glucopyranosyl. In the HMBC spectrum, the long-range correlations from $\mathrm{H}-1^{\prime \prime \prime}$ to $\mathrm{C}-6^{\prime} ; \mathrm{H}-1^{\prime \prime}$ to $\mathrm{C}-2^{\prime} ; \mathrm{H}-1^{\prime}$ to $\mathrm{C}-9$ (Figure 4 ) were observed. Moreover, treated 8 with $1 \mathrm{M} \mathrm{HCl}$, D-glucose was yielded [28]. Consequently, the structure of mongophenoside $\mathrm{A}_{2}$ (8) was elucidated.

The ESI-Q-Orbitrap MS spectrum of mongophenoside $\mathrm{A}_{3}$ (9) displayed the same molecular formula, $\mathrm{C}_{27} \mathrm{H}_{38} \mathrm{O}_{19}\left(\mathrm{~m} / \mathrm{z} 665.19452[\mathrm{M}-\mathrm{H}]^{-}\right.$; calculated for $\left.\mathrm{C}_{27} \mathrm{H}_{37} \mathrm{O}_{19}, 665.19236\right)$ as that of 8 . Meanwhile, the ${ }^{1} \mathrm{H},{ }^{13} \mathrm{C}$ NMR (Table 2) and 2D NMR $\left({ }^{1} \mathrm{H}^{1} \mathrm{H}\right.$ COSY, HSQC, HMBC, HSQC-TOCSY) spectra suggested they had same functional groups as following: trans-caffeic acid aglycone [ $\delta 6.45$ $(1 \mathrm{H}, \mathrm{d}, J=16.0 \mathrm{~Hz}, \mathrm{H}-8), 7.19$ (1H, br. s, H-2), 7.12 (2H, m, H-5 and H-6), 7.61 (1H, d, J = 16.0 Hz, H-7)] and three $\beta$-D-glucopyranosyl groups $\left[\delta 4.43\left(1 \mathrm{H}, \mathrm{d}, J=7.5 \mathrm{~Hz}, \mathrm{H}-1^{\prime \prime \prime}\right), 4.80\left(1 \mathrm{H}, \mathrm{d}, J=7.5 \mathrm{~Hz}, \mathrm{H}-1^{\prime}\right)\right.$, $\left.5.57\left(1 \mathrm{H}, \mathrm{d}, J=7.0 \mathrm{~Hz}, \mathrm{H}-1^{\prime \prime}\right)\right]$. Finally, the connectivities of the above-mentioned groups were clarified by the long-range correlations from $\mathrm{H}-1^{\prime}$ to $\mathrm{C}-4 ; \mathrm{H}-1^{\prime \prime}$ to $\mathrm{C}-9 ; \mathrm{H}-1^{\prime \prime \prime}$ to $\mathrm{C}-2^{\prime \prime}$ (Figure 4 ), as shown in its HMBC experiment.

Mongophenoside B (10) was obtained as a white powder with positive optical rotation $\left([\alpha]_{\mathrm{D}}{ }^{25}\right.$ +8.0, MeOH). Its ESI-Q-Orbitrap MS spectrum showed the negative ion peak at $m / z 517.15668$ [M $\mathrm{H}]^{-}$(calculated for $\mathrm{C}_{22} \mathrm{H}_{29} \mathrm{O}_{14}, 517.15518$ ), which indicated the molecular formula of it was $\mathrm{C}_{22} \mathrm{H}_{30} \mathrm{O}_{14}$. Acid hydrolysis 10 with $1 \mathrm{M} \mathrm{HCl}$, D-glucose was liberated [28]. The ${ }^{1} \mathrm{H},{ }^{13} \mathrm{C}$ NMR (Table 2) and 2D NMR spectra of it suggested the existence of one trans-feruloyl $[\delta 6.34(1 \mathrm{H}, \mathrm{d}, J=16.0 \mathrm{~Hz}, \mathrm{H}-8)$, $6.80(1 \mathrm{H}, \mathrm{d}, J=8.0 \mathrm{~Hz}, \mathrm{H}-5), 7.06(1 \mathrm{H}, \mathrm{br} . \mathrm{d}$, ca. $J=8 \mathrm{~Hz}, \mathrm{H}-6), 7.17(1 \mathrm{H}$, br. s, H-2), $7.60(1 \mathrm{H}, \mathrm{d}$, $\left.J=16.0 \mathrm{~Hz}, \mathrm{H}-7), 3.88\left(3 \mathrm{H}, \mathrm{s}, 3-\mathrm{OCH}_{3}\right)\right]$, one $\beta$-D-glucopyranosyl [ $\left.\delta 4.48\left(1 \mathrm{H}, \mathrm{d}, J=8.0 \mathrm{~Hz}, \mathrm{H}-1^{\prime}\right)\right]$, together with one $\alpha$-D-glucopyranosyl $\left[\delta 5.10\left(1 \mathrm{H}, J=3.5 \mathrm{~Hz}, \mathrm{H}-1^{\prime \prime}\right)\right]$. Moreover, the long-range correlations from $\mathrm{H}-1^{\prime}$ to $\mathrm{C}-9 ; \mathrm{H}-1^{\prime \prime}$ to $\mathrm{C}-2^{\prime}$ were observed in its HMBC experiment. On the basis of above-mentioned evidence, the structure of mongophenoside B (10) was identified as trans-ferulic acid-9-O- $\alpha$-D-glucopyranosyl $(1 \rightarrow 2)-\beta$-D-glucopyranoside.
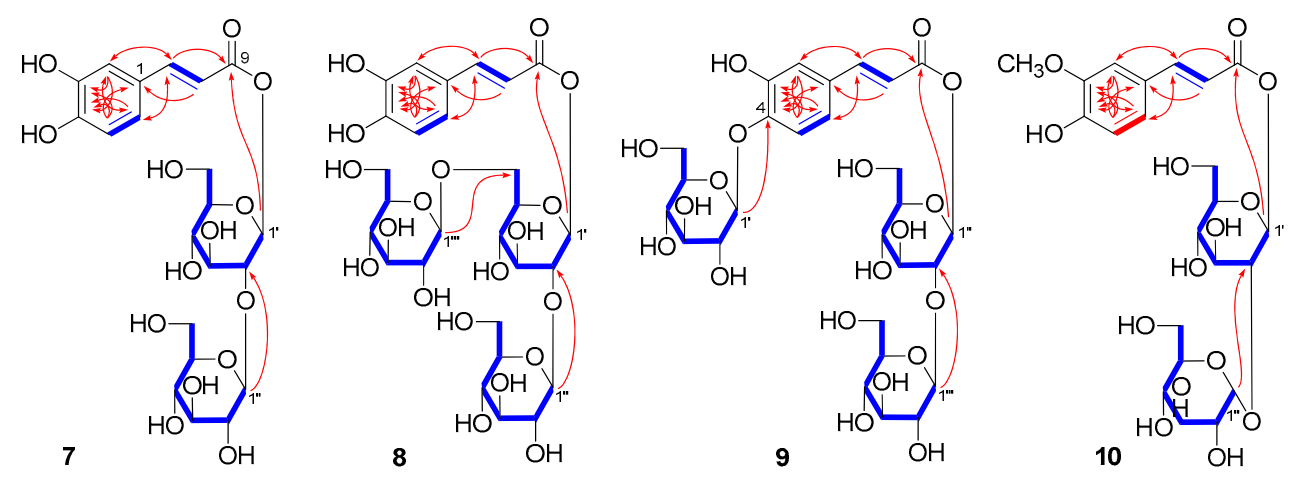

Figure 4. Key ${ }^{1} \mathrm{H}^{1} \mathrm{H}$ COSY and HMBC correlations of 7-10.

\subsection{Inhibitory Effects of Obtained Compounds 1-31 on the Motility of Mouse Isolated Intestine Tissue}

Moreover, the obtained constituents of 1-31 were tested for frequency and height by using a tissue perfusion method [30]. Through tissue perfusion experiments, it was found that all compounds displayed no effect on isolated intestinal tissue contraction frequency (Table 3). While almost all isolates exhibited the tendency of increasing the contraction amplitude of mouse small intestinal muscle though only flavonoids 3, 4, 11-15, 21-23, and 26, as well as phenolic acids 7, 29, and 30 showed significant difference comparing with normal group. 
Table 3. Inhibitory effects of compounds 1-31 on motility of mouse isolated intestine tissue.

\begin{tabular}{cccccc}
\hline \multirow{2}{*}{ Compd. } & \multicolumn{2}{c}{ Intestine Motility (\%) } & \multirow{2}{*}{ Compd. } & \multicolumn{2}{c}{ Intestine Motility (\%) } \\
\cline { 2 - 3 } \cline { 5 - 6 } & Relative Height & Relative Frequency & & Relative Height & Relative Frequency \\
\hline $\mathrm{N}$ & $100.0 \pm 4.9$ & $100.0 \pm 1.2$ & $\mathbf{1 6}$ & $116.1 \pm 10.4$ & $81.3 \pm 11.1$ \\
$\mathrm{P}$ & $190.8 \pm 19.2^{* *}$ & $85.6 \pm 2.6$ & $\mathbf{1 7}$ & $108.1 \pm 6.1$ & $97.3 \pm 2.3$ \\
$\mathbf{1}$ & $112.3 \pm 2.3$ & $99.4 \pm 1.2$ & $\mathbf{1 8}$ & $116.6 \pm 4.4$ & $106.6 \pm 4.5$ \\
$\mathbf{2}$ & $105.1 \pm 19.5$ & $98.8 \pm 2.9$ & $\mathbf{1 9}$ & $107.7 \pm 3.1$ & $99.3 \pm 3.5$ \\
$\mathbf{3}$ & $148.9 \pm 4.5^{* *}$ & $100.8 \pm 0.9$ & $\mathbf{2 0}$ & $107.8 \pm 32.7$ & $99.4 \pm 0.6$ \\
$\mathbf{4}$ & $170.0 \pm 6.4^{*}$ & $98.6 \pm 2.1$ & $\mathbf{2 1}$ & $121.9 \pm 6.6^{*}$ & $95.2 \pm 4.4$ \\
$\mathbf{5}$ & $99.7 \pm 25.0$ & $101.6 \pm 7.7$ & $\mathbf{2 2}$ & $125.2 \pm 8.1^{*}$ & $97.4 \pm 4.0$ \\
$\mathbf{6}$ & $107.9 \pm 18.9$ & $96.7 \pm 3.1$ & $\mathbf{2 3}$ & $142.2 \pm 11.2^{*}$ & $101.1 \pm 3.7$ \\
$\mathbf{7}$ & $157.4 \pm 20.8^{*}$ & $94.9 \pm 2.7$ & $\mathbf{2 4}$ & $117.9 \pm 12.7$ & $98.3 \pm 2.8$ \\
$\mathbf{8}$ & $150.5 \pm 25.9$ & $95.6 \pm 2.8$ & $\mathbf{2 5}$ & $104.9 \pm 7.7$ & $96.3 \pm 1.9$ \\
$\mathbf{9}$ & $149.1 \pm 36.2$ & $94.6 \pm 3.8$ & $\mathbf{2 6}$ & $137.4 \pm 2.4^{* *}$ & $96.2 \pm 2.8$ \\
$\mathbf{1 0}$ & $121.1 \pm 21.6$ & $98.7 \pm 3.0$ & $\mathbf{2 7}$ & $105.6 \pm 32.3$ & $91.8 \pm 3.8$ \\
$\mathbf{1 1}$ & $123.2 \pm 6.8^{*}$ & $99.1 \pm 7.1$ & $\mathbf{2 8}$ & $136.8 \pm 12.4$ & $97.1 \pm 2.0$ \\
$\mathbf{1 2}$ & $144.2 \pm 14.3^{*}$ & $95.7 \pm 1.3$ & $\mathbf{2 9}$ & $148.1 \pm 6.8^{*}$ & $105.7 \pm 4.1$ \\
$\mathbf{1 3}$ & $151.5 \pm 17.1^{*}$ & $98.8 \pm 4.4$ & $\mathbf{3 0}$ & $125.0 \pm 1.6^{* *}$ & $98.8 \pm 2.3$ \\
$\mathbf{1 4}$ & $120.4 \pm 9.2^{*}$ & $98.4 \pm 1.7$ & $\mathbf{3 1}$ & $97.8 \pm 1.9$ & $98.8 \pm 1.2$ \\
$\mathbf{1 5}$ & $143.7 \pm 1.3^{* *}$ & $100.6 \pm 3.1$ & & & \\
\hline
\end{tabular}

Values are the means \pm standard error of measurement, significantly different from the control group ${ }^{*} p<0.05$, $* * p<0.01, \mathrm{n}=6$. Normal $(\mathrm{N})$ : isolated intestine tissue; Positive control $(\mathrm{P})$ : Mosapride citrate dihydrate, final concentration was $200 \mu \mathrm{g} / \mathrm{mL}$. Compounds 1-31: final concentration was $50 \mu \mathrm{M}$. Frequency, and height of normal group was set as $100 \%$, relative values of them were calculated as: (sample/normal) $\times 100 \%$.

\subsection{Qualitative Analysis}

As an important edible medicinal plant for Mongolian people, A. mongolicum has made a great contribution to the development of the local economy, yet there is a lack of analysis of its quality until now.

Our systematic phytochemistry isolation results indicated the main constituents of AM were flavonoids and phenolic acids. The aglycones in the plant mainly included quercetin, kaempferol, as well as isorhamnetin for flavonoid glycosides; while coumaric acid, caffeic acid, and ferulic acid for phenolic acid glycosides. The sugars consisted of $\beta$-D-glucopyranoside (Glc), $\alpha$-D-glucopyranoside ( $\alpha$-Glc), $\beta$-D-glucuronic acid (Glu), and $\alpha$-L-rhamnopyranoside (Rha). While $\alpha$-Glc was only found in phenolic acid glycosides, Glu and Rha substituted only for flavonoid glycosides.

As for flavonoids, 3-, 7-, and 4'-OH of quercetin, kaempferol, and isorhamnetin were easily substituted by various glycosyls to format $\mathrm{O}$-glycosides. Among them, 7- and 4'-OH was substituted by monosaccharose such as Glc and Glu, while Glu was found to only link with their 7-position. Meanwhile, 3-OH was with a high degree of glycosylation, having one to three sugar moieties, and all of the glycosyl groups directly linked to flavonoid was Glc group, then its 2-, 4-, or 6-position was substituted by another Glc continuously; moreover, its 6-position could also be replaced by Rha [to form rutinosyl (Rut)] or acetyl group (Figure 5).

On the other hand, the carboxyl of obtained phenolic acids from AM was easily substituted by sugar moiety such as $\operatorname{Glc}(1 \rightarrow 2) G l_{c}-, \alpha-G l_{c}(1 \rightarrow 2) G l_{c}-$, or Glc $(1 \rightarrow 6) \operatorname{Glc}(1 \rightarrow 2)$ Glc- on their 9-position, while $4-\mathrm{OH}$ of them was only substituted by monosaccharose, Glc (Figure 6).

Herein, on the basis of above-mentioned phytochemistry study, a fast analysis method for flavonoids and phenolic acids in AM was established by LC-MS on an ESI-Q-Orbitrap MS in negative ion mode (Figure 7). According to the chromatographic retention time $\left(t_{R}\right)$ and the exact mass-to-charge ratio $(\mathrm{m} / \mathrm{z}), 31$ compounds (1-31) were unambiguously identified by comparing to the standard references. Meanwhile, the rules of the MS/MS fragmentation pattern and chromatographic elution order have been generalized. Then, five flavonoid glycosides (32-36) and one phenolic acid glycoside (37) were tentatively speculated. Among them, $\mathbf{3 6}$ was a potential new compound (Table S1, Figure 8). 


\section{Aglycone}<smiles>O=c1c(O)c(-c2ccc(O)cc2)oc2cc(O)cc(O)c12</smiles>

Kaempferol<smiles>O=c1c(O)c(-c2ccc(O)c(O)c2)oc2cc(O)cc(O)c12</smiles>

Quercetin<smiles>COc1cc(-c2oc3cc(O)cc(O)c3c(=O)c2O)ccc1O</smiles>

Isorhamnetin

Sugar moiety

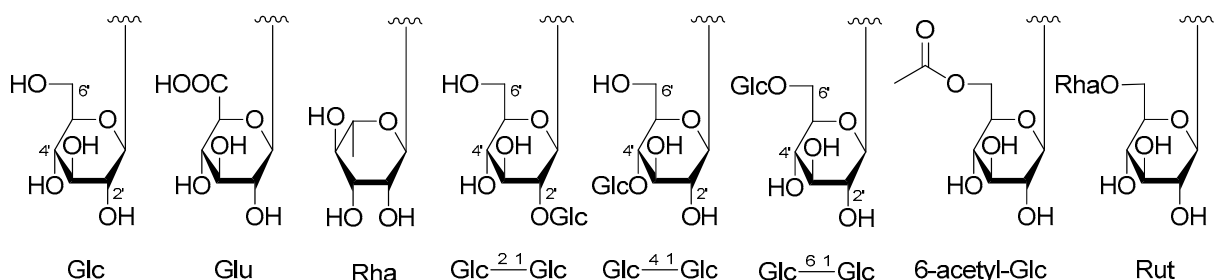

Figure 5. The structure of aglycones and glycosyls of flavonoids from the aerial parts of A. mongolicum.

\section{Aglycone}<smiles>O=C(O)/C=C/c1ccc(O)cc1</smiles>

Sugar moiety

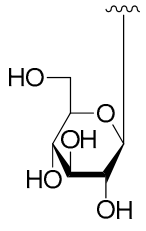

Glc<smiles>O=C(O)/C=C/c1ccc(O)c(O)c1</smiles>

Caffeic acid

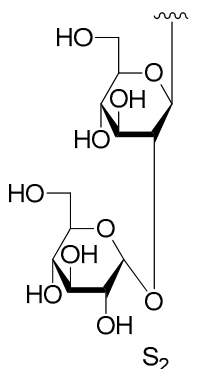<smiles>COc1cc(/C=C/C(=O)O[Na])ccc1O</smiles>

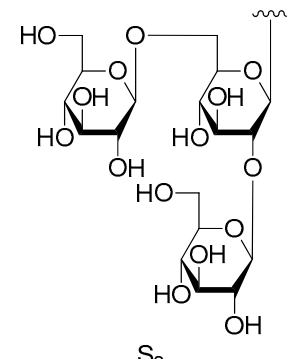

Figure 6. The structure of aglycones and glycosyls of phenolic acids from the aerial parts of A. mongolicum. 

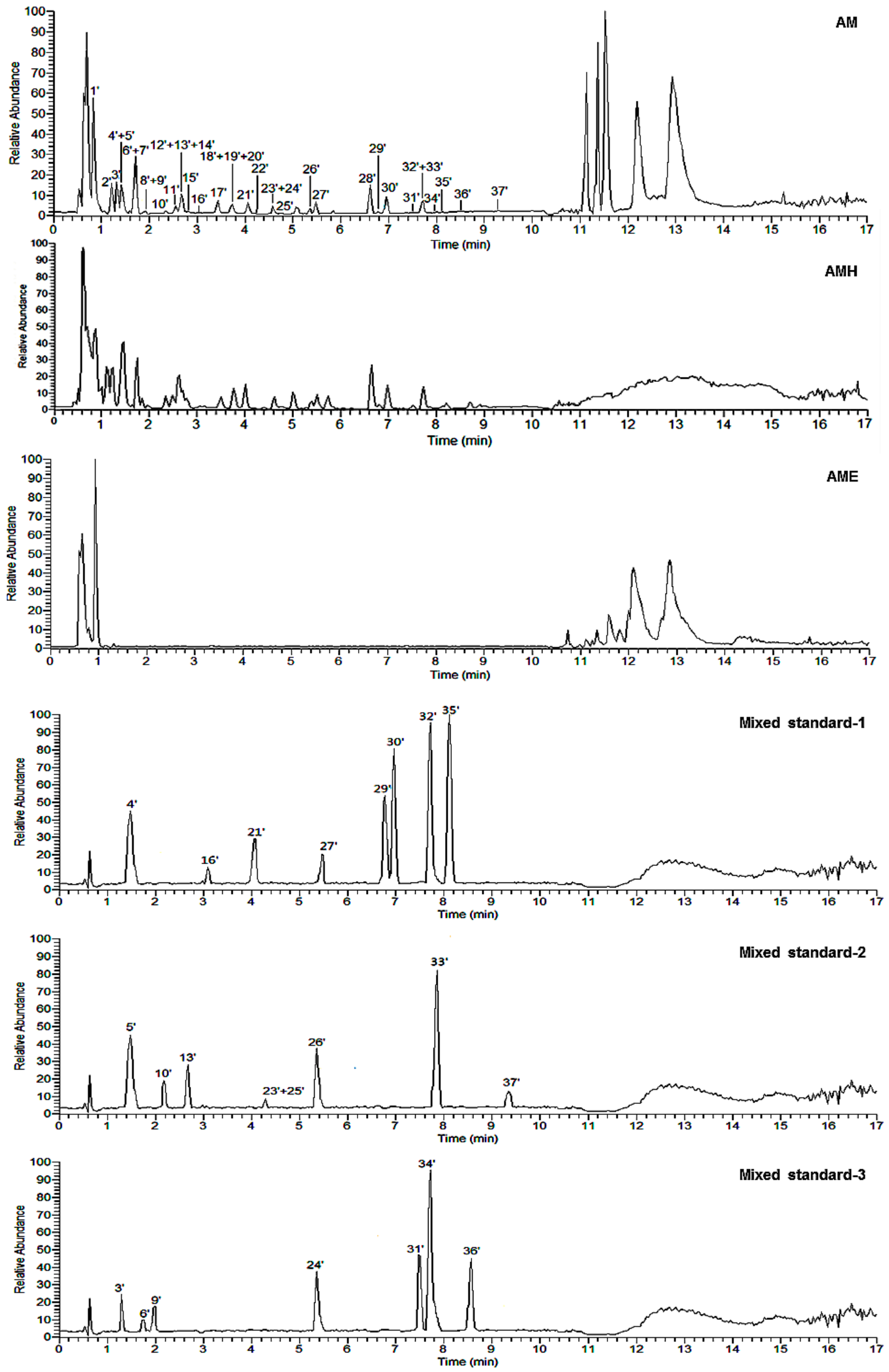

Figure 7. Cont. 

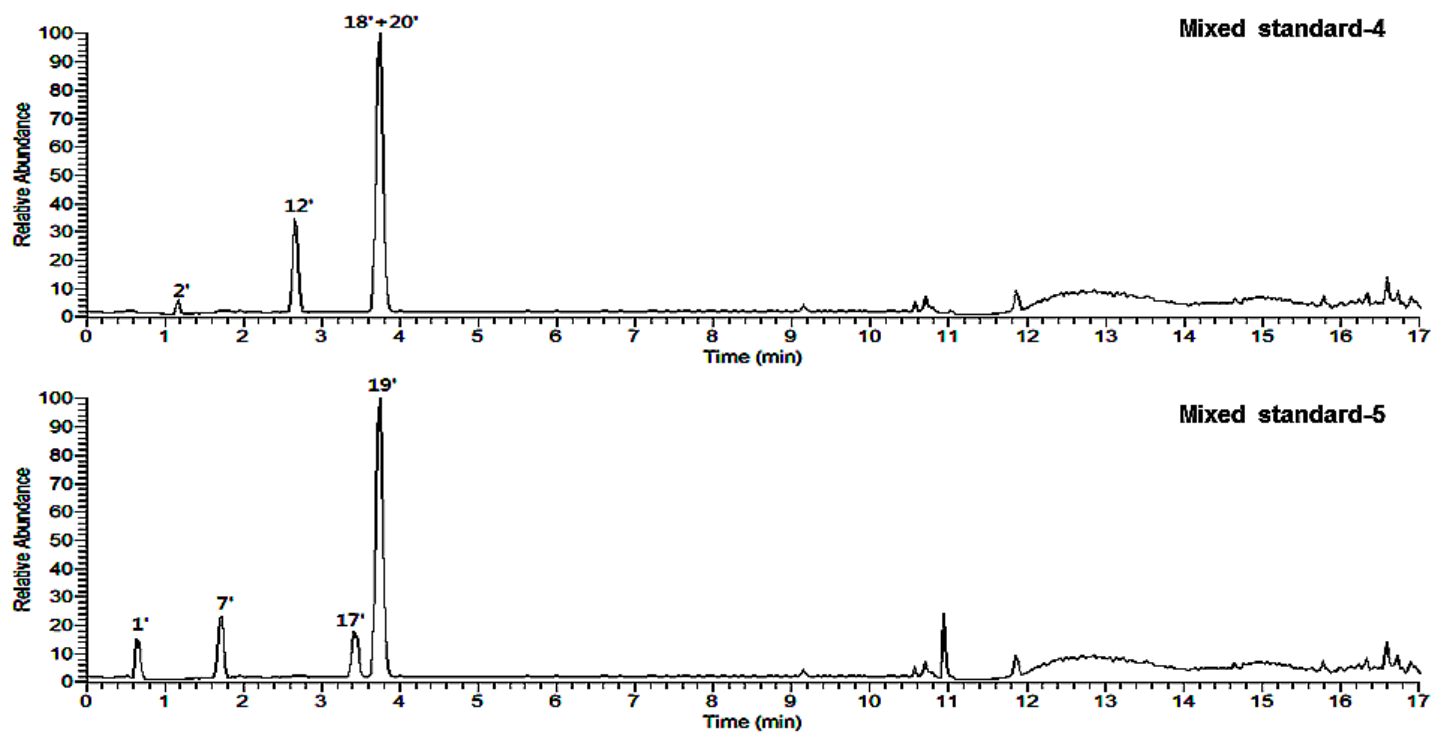

Figure 7. Base peak chromatograms of $\mathrm{AM}$ (the extract obtained from fresh the aerial parts of A. mongolicum heated reflux with $95 \% \mathrm{EtOH}$ and $50 \% \mathrm{EtOH}$ one time each, successively), $\mathrm{AMH}\left(\mathrm{H}_{2} \mathrm{O}\right.$ layer extract), AME (EtOAc layer extract), and mixed standard references.<smiles>[R6]OC(=O)/C=C/c1ccc(O[R20])c([R])c1</smiles>

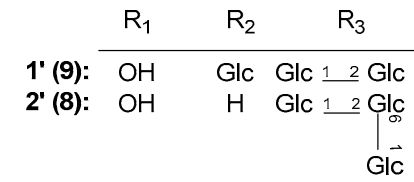

7' (7): $\mathrm{OH} \quad \mathrm{H} \quad \mathrm{Glc} 12 \mathrm{Glc}$ 12' (28): H H Glc 12 Glc 17' (29): $\mathrm{OCH}_{3}$ H Glc $12 \mathrm{Glc}$ 18' (10): $\mathrm{OCH}_{3} \quad \mathrm{H} \alpha$-Glc $\frac{12}{2} \mathrm{Glc}$ 19' (30): $\mathrm{OH} \quad \mathrm{H} \quad \mathrm{H}$<smiles>O=C(/C=C\c1ccc(O)cc1)O[Ge]Cl</smiles>

15' (35)<smiles>COCc1ccccc1</smiles><smiles>[R6]Oc1ccc(-c2oc3cc(O[R6])cc(O)c3c(=O)c2O)cc1</smiles><smiles>[R20]Oc1cc(O)c2c(=O)c(O[R10])c(-c3ccc([R20])c(O[R20])c3)oc2c1</smiles>

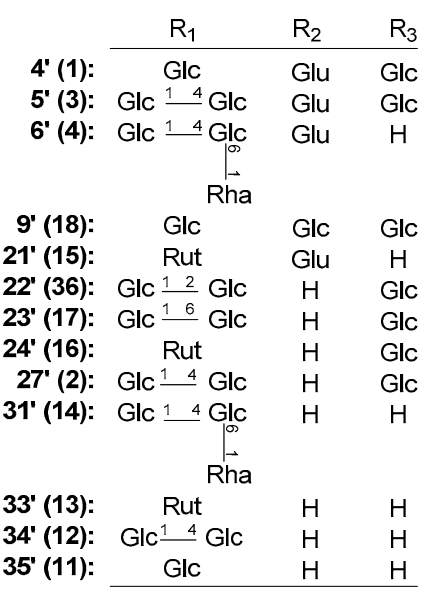

\begin{tabular}{|c|c|c|c|c|}
\hline & $\mathrm{R}_{1}$ & $\mathrm{R}_{2}$ & $\mathrm{R}_{3}$ & $\mathrm{R}_{4}$ \\
\hline $3^{\prime}(6)$ : & Glc & Glu & $\mathrm{H}$ & Glc \\
\hline 8' (32): & Glc $\frac{12}{2}$ Glc & Glu & $\mathrm{H}$ & $\mathrm{H}$ \\
\hline $10^{\prime}(26):$ & Glc & Glc & $\mathrm{H}$ & Glc \\
\hline 11' (33): & Glc $\frac{16}{6} \mathrm{Glc}$ & Glu & $\mathrm{H}$ & $\mathrm{H}$ \\
\hline 13' (25): & Rut & Glu & $\mathrm{H}$ & $\mathrm{H}$ \\
\hline 14' (34): & Glc $\frac{14}{4} \mathrm{Glc}$ & Glu & $\mathrm{H}$ & $\mathrm{H}$ \\
\hline $16^{\prime}(23):$ & Glc & $\mathrm{H}$ & $\mathrm{H}$ & Glc \\
\hline $25^{\prime}(24):$ & Rut & Glc & $\mathrm{H}$ & $\mathrm{H}$ \\
\hline 26' (22): & Rut & $\mathrm{H}$ & $\mathrm{H}$ & $\mathrm{H}$ \\
\hline 28' (37): & Rut & $\mathrm{H}$ & $\mathrm{CH}_{3}$ & $\mathrm{H}$ \\
\hline 29' (21): & Glc 14 Glc & $\mathrm{H}$ & $\mathrm{H}$ & $\mathrm{H}$ \\
\hline 30' (19): & Glc & $\mathrm{H}$ & $\mathrm{H}$ & $\mathrm{H}$ \\
\hline $32^{\prime}(5):$ & Glc ${ }^{1}-4\left(6^{\prime \prime}-\right.$ acetyl $)-C$ & $\mathrm{CH}$ & $\mathrm{H}$ & $\mathrm{H}$ \\
\hline 36' (20): & (6"-acetyl)-GIc & $\mathrm{H}$ & $\mathrm{H}$ & $\mathrm{H}$ \\
\hline 37' (27): & Glc & $\mathrm{H}$ & $\mathrm{CH}_{3}$ & $\mathrm{H}$ \\
\hline
\end{tabular}

Figure 8. The structures of tentatively presumed compounds from the aerial parts of A. mongolicum.

\subsubsection{Structural Elucidation of Flavonoids}

Peaks $3^{\prime}-6^{\prime}, 9^{\prime}, 10^{\prime}, 13^{\prime}, 16^{\prime}, 21^{\prime}, 23^{\prime}-27^{\prime}$ and $29^{\prime}-37^{\prime}$ were identified by comparison with reference standards (Table S1, Figure 7).

Figure 9 and Figure S74 showed the MS/MS fragmentation pattern of flavonoid glycosides with kaempferol and quercetin aglycones, which suggested both of two kinds of flavonoid glycosides could be ionized to generate heterolytic cleavage with fragments ion peak at $m / z 285.03936\left(\mathrm{Y}_{\mathrm{K} 0}{ }^{-}\right)$ for kaempferol and $m / z 301.03428\left(\mathrm{Y}_{\mathrm{Q}^{-}}{ }^{-}\right.$) for quercetin, as well as hemolytic cleavage with fragments ion peak at $m / z 284.03154\left[\mathrm{Y}_{\mathrm{K} 0}{ }^{-}-\mathrm{H}\right]^{-}$for kaempferol and $m / z 300.02645\left[\mathrm{Y}_{\mathrm{Q}{ }^{-}}-\mathrm{H}\right]^{-}$for quercetin, respectively. Then, kaempferol aglycone could be further cleavage to generate fragment ion peaks at $m / z 255.02880,179.02371$, and 151.00259. The fragment ion peaks at $m / z 271.02371,255.02880$, 243.02880, 179.02371, as well as 151.00259, were yielded from quercetin by a series of reactions 
including decarbonylation, dehydrogenation, retro Diels-Alder reaction, and the reaction to remove the $\mathrm{B}$ ring. The above-mentioned characteristic fragment ions could be used to distinguish the type of aglycone.

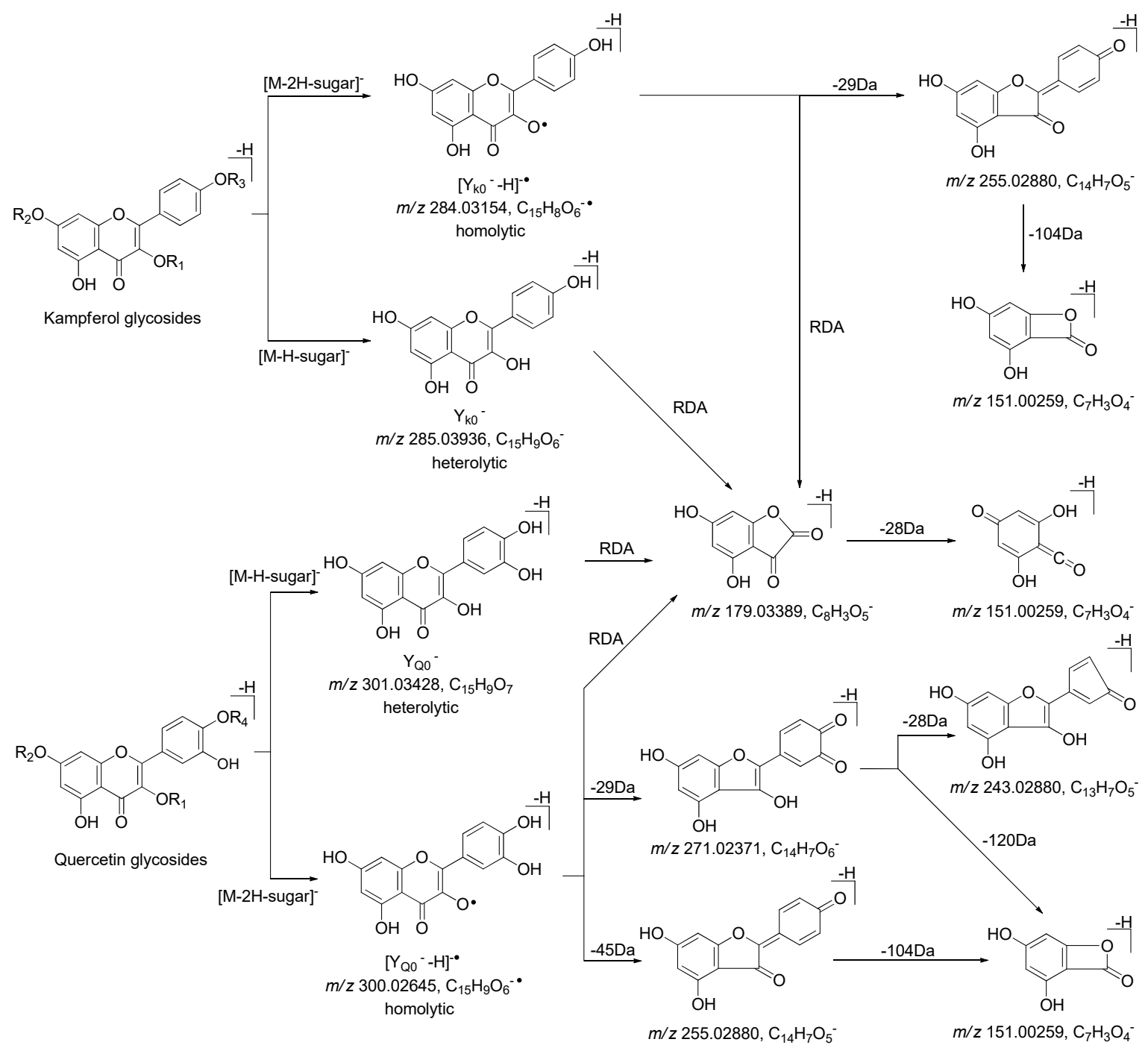

Figure 9. The proposed fragmentation pathways of kaempferol and quercetin glycosides.

Meanwhile, when the $4^{\prime}$-position of flavanol aglycone was glycosylated to format $O$-glycoside, the debris ions peaks $\left(\left[\mathrm{Y}_{\mathrm{K} 0}{ }^{-} 2 \mathrm{H}\right]^{-}\right)$at $m / z 283.02371$ for kaempferol and $m / z 299.01863\left(\left[\mathrm{Y}_{\mathrm{Q} 0}{ }^{-} 2 \mathrm{H}^{-}\right)\right.$for quercetin glycosides were stronger than those of $m / z 284.03154$ and 300.02645, respectively (Table S1). Therefore, their ionic strength could be used to quickly determine whether the C-4' position of the aglycone was replaced by sugar.

Peaks $9^{\prime}, 22^{\prime}, 23^{\prime}$, and $27^{\prime}$ were obtained by extracting ion of $\mathrm{m} / \mathrm{z} 771.19783$ from the total ion chromatogram of AM (Figure 10), among them, 9', 23', and 27' were clarified to be kaempferol-3,7,4'-tri-O- $\beta$-glucoside (18), kaempferol-3-O-gentiobioside-4' -O-glucopyranoside (17), and mongoflavonoside $\mathrm{A}_{2}$ (2) by comparing with reference standards. Then, according to the above-mentioned biosynthetic pathway of substituted sugar, peak 22' was tentatively presumed to be kaempferol-3-O- $\beta$-D-glucopyranosyl $(1 \rightarrow 2)-O-\beta$-D-glucopyranosyl-4'-O- $\beta$-D-glucopyranoside (36), which was one new compound. 


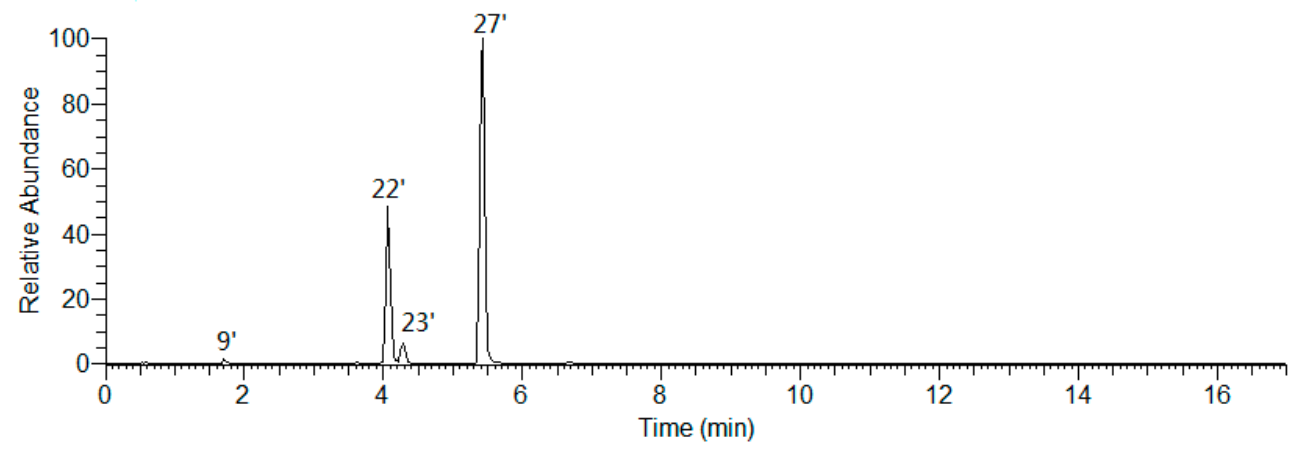

Figure 10. The EIC of the $m / z 771.19783$.

Moreover, during the comparison of the chromatographic retention behavior of peaks $9^{\prime}, 22^{\prime}$, $23^{\prime}$, and $27^{\prime}$, we discovered the effect of sugar substitution position on $t_{\mathrm{R}}$ was 3,7,4'-tri-O-Glc < 3-O-Glc $(1 \rightarrow 2)-G l c-4^{\prime}-O-G l c<3-O-G l c(1 \rightarrow 6)$-Glc-4'-O-Glc $<3-O-G l c(1 \rightarrow 4)-G l c-4^{\prime}-O-G l c$.

The molecular formula of peaks $3^{\prime}\left(\mathrm{m} / \mathrm{z}\right.$ 801.17407), $8^{\prime}(\mathrm{m} / \mathrm{z} 801.17462), 11^{\prime}(\mathrm{m} / \mathrm{z} 801.17389)$, and $14^{\prime}(\mathrm{m} / \mathrm{z}$ 801.17200) were all $\mathrm{C}_{33} \mathrm{H}_{38} \mathrm{O}_{23}$ (Figure 11). Peak $3^{\prime}$ was unambiguously identified as mongoflavonoside $\mathrm{B}_{2}$ (6) by comparison with reference standard. According to the MS/MS fragment ion peaks at $m / z 301.03428$, $300.02645,299.01863,271.02371,255.02880$, and 151.00259 , peaks $8^{\prime}, 11^{\prime}$, and $14^{\prime}$ were deduced to be with quercetin aglycone. On the other hand, the fragment ion peaks at $m / z 625.13993[\mathrm{M}-\mathrm{H}-176]^{-}, 301.03428$ $[\mathrm{M}-\mathrm{H}-176-162-162]^{-}$suggested the presences of one $\beta$-D-Glu and two $\beta$-D-Glc in them. Since the strength of fragment ion peak at $m / z 299.01863$ was weaker than that of $m / z 300.02645$, we could propose that $4^{\prime}-\mathrm{OH}$ of quercetin was not be glycosidated. According to the above-mentioned biosynthetic pathway of substituted sugar and effect of sugar substitution position on $t_{\mathrm{R}}$, peaks $8^{\prime}, 11^{\prime}$, and $14^{\prime}$ were tentatively presumed to be quercetin-3-O- $\beta$-D-glucopyranosyl $(1 \rightarrow 2)-\beta-\mathrm{D}$-glucopyranosyl-7-O- $\beta$-D-glucuronide (32), $\quad$ quercetin-3-O- $\beta$-D-glucopyranosyl $(1 \rightarrow 6)-\beta$-D-glucopyranosyl-7-O- $\beta$-D-glucuronide and quercetin-3-O- $\beta$-D-glucopyranosyl $(1 \rightarrow 4)-\beta$-D-glucopyranosyl-7-O- $\beta$-D-glucuronide (34), respectively.

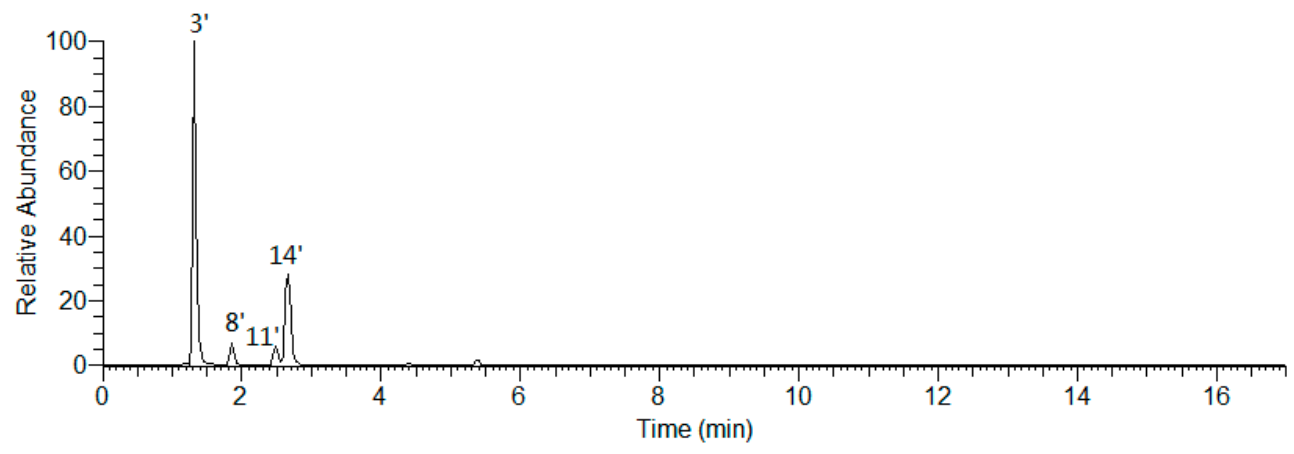

Figure 11. The EIC of the $m / z$ 801.17201.

The molecular formula of peak $28^{\prime}(\mathrm{m} / \mathrm{z} 799.19391)$ was $\mathrm{C}_{33} \mathrm{H}_{38} \mathrm{O}_{23}$. Its MS/MS fragment ion peaks displayed at $m / z 623.15869$ [M - H - 176], $315.05048[\mathrm{M}-\mathrm{H}-176-162-146]^{-}, 300.02713,271.02469$, and 243.02880 suggested the aglycone of it was isorhamnetin and the substituted sugar moieties included one Glu, one Glc, and one Rha. According to the biosynthesis laws summarized above, peak $28^{\prime}$ was deduced to be isorhamnetin-3-O-rutinosyl-7-O- $\beta$-D-glucuronide (37) (Table S1, Figure S75).

\subsubsection{Structural Elucidation of Phenolic Acids}

Peaks $1^{\prime}, 2^{\prime}, 7^{\prime}, 12^{\prime}$, and $17^{\prime}-20^{\prime}$ were identified unequivocally by comparing with reference standards (Table S1, Figure 7). As what have been mentioned above, the aglycones of phenolic acid glycosides included coumaric acid, caffeic acid, and ferulic acid. It was well known that the characteristic ions of coumaroyl, caffeoyl, and feruloyl were at $\mathrm{m} / \mathrm{z} 163.03897\left(\left[\right.\right.$ coumaroyl $-\mathrm{H}^{-}$), 
$179.03389\left([\text { caffeoyl }-\mathrm{H}]^{-}\right)$, and $193.04954\left([\text { feruloyl }-\mathrm{H}]^{-}\right)$, respectively [31]. Then, all of the ions would further generate fragment ion peaks (as shown in Figure 12) by removing $44 \mathrm{Da}\left(-\mathrm{CO}_{2}\right), 28 \mathrm{Da}$ $(-\mathrm{CO})$, and $18 \mathrm{Da}\left(-\mathrm{H}_{2} \mathrm{O}\right)$, respectively.

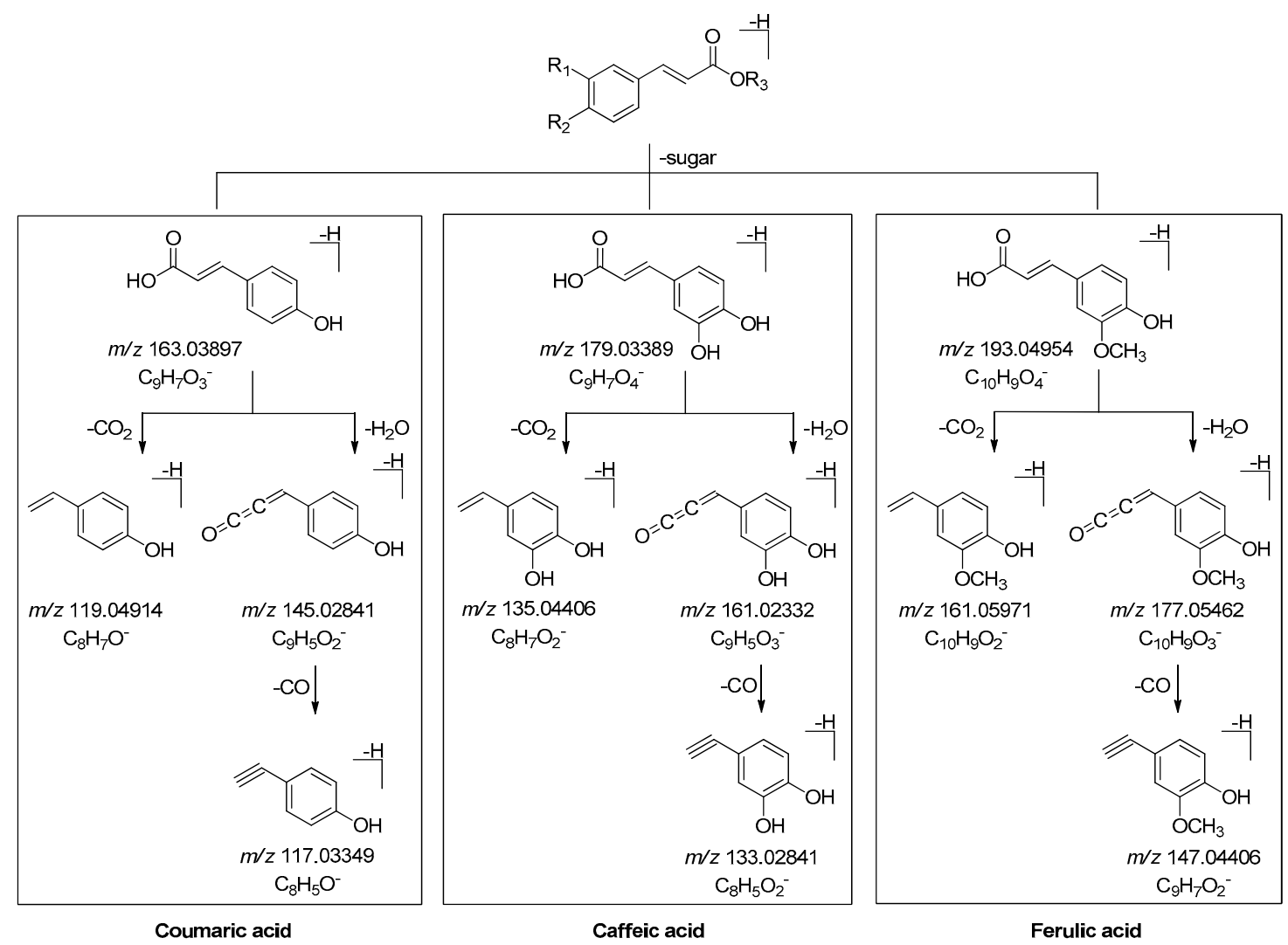

Figure 12. The proposed fragmentation pathways of coumaric acid, caffeic acid, and ferulic acid glycosides.

Meanwhile, the phenomenon of the neutral loss 120 Da on the basis of $[\mathrm{M}-\mathrm{H}]^{-}$were only found in $\beta$-D-glucopyranosyl $(1 \rightarrow 2)$ - $\beta$-D-glucopyranosyl substituted phenolic acid glycosides 7 (peak $7^{\prime}$ ), 28 (peak 12'), and 29 (peak 17') (Figure 13 and Figure S76), which could be used to distinguish the type of substituted sugar moieties.
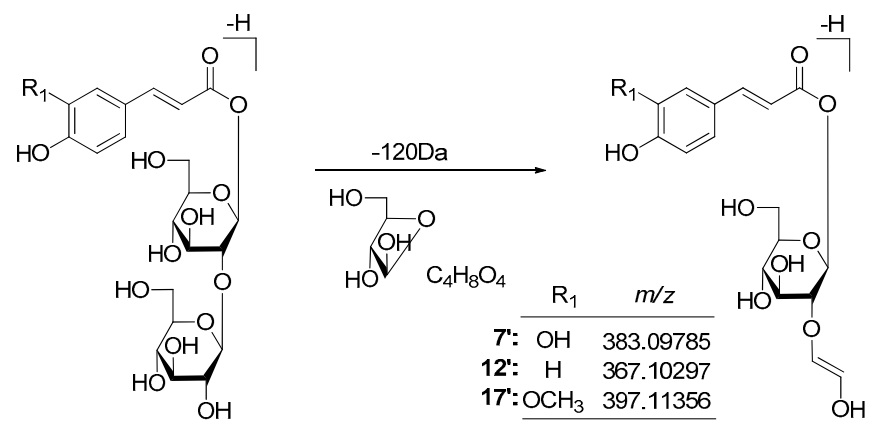

Figure 13. The proposed fragmentation pathways of $\beta$-D-glucopyranosyl $(1 \rightarrow 2)-\beta$-D-glucopyranosyl substituted phenolic acid glycosides.

Moreover, comparing the $t_{\mathrm{R}}$ of compounds 10 (peak 18') and 29 (peak 17'), $\alpha$-D-glucopyranosyl-substituted phenolic acid glycoside was found to have the shorter $t_{R}$ than that of $\beta$-D-glucopyranosyl-substituted ones. 
The molecular formula of peak $15^{\prime}\left(\mathrm{m} / z\right.$ 487.14313) was $\mathrm{C}_{21} \mathrm{H}_{28} \mathrm{O}_{13}$. Its MS/MS fragment ion peaks displayed at $m / z$ 367.10297, 163.03888, and 145.02829, which was similar to those of peak 12' (Table S1, Figure 14). According to the above-mentioned chromatographic retention behavior, we could deduce that peak $15^{\prime}$ was not $p$-hydroxycinnamic acid-9-O- $\alpha$-D-glucopyranosyl $(1 \rightarrow 2)$ - $\beta$-D-glucopyranoside. As Han et al. reported, the $t_{\mathrm{R}}$ of cis-phenylpropane glycoside was longer than that of trans one when they were analysed by HPLC with the acetonitrile-water system [24]. Consequently, peak 15' was tentatively presumed to be cis- $p$-hydroxycinnamate sophorose (35).
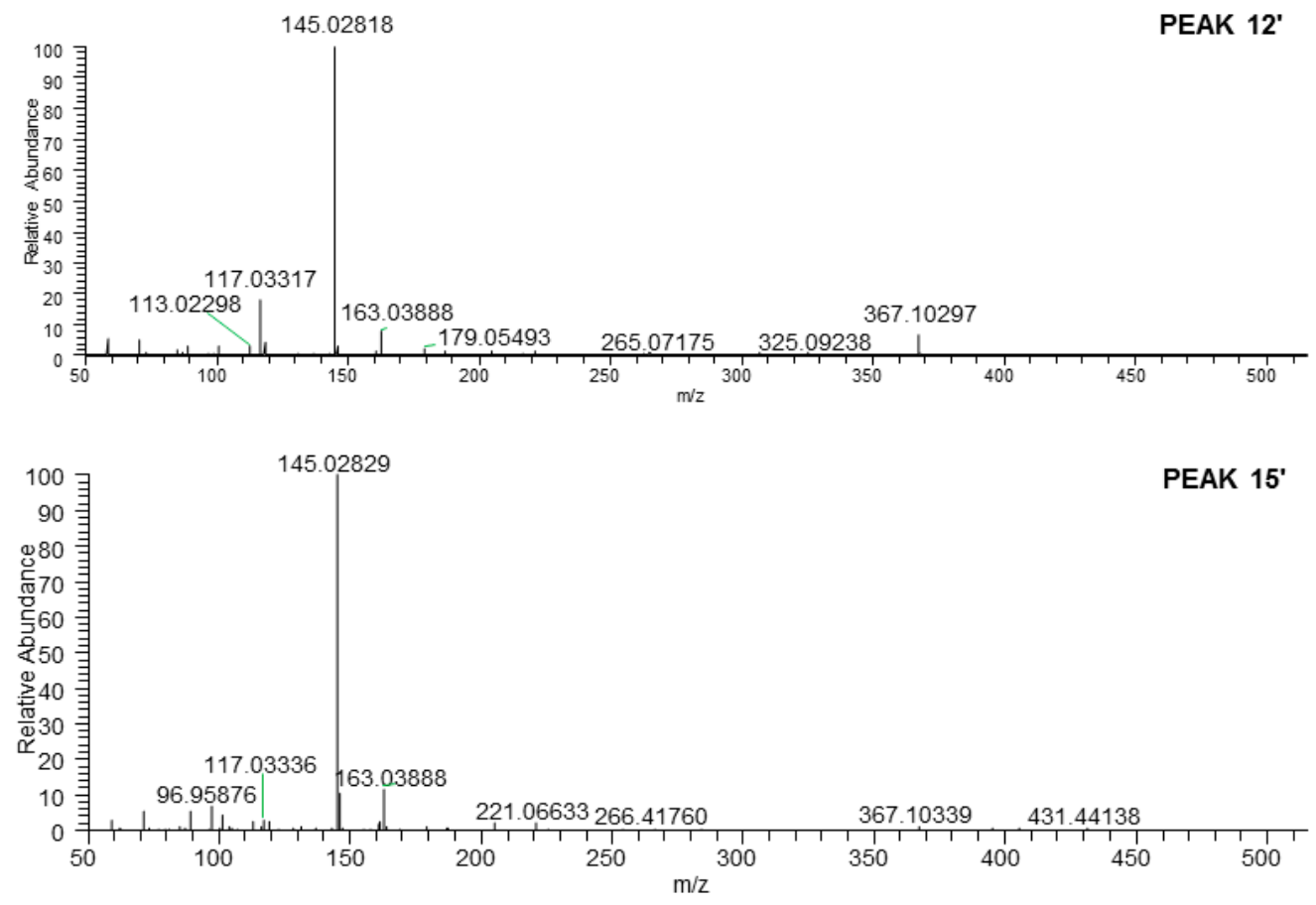

Figure 14. The tandem MS of the $[\mathrm{M}-\mathrm{H}]^{-}$ions for peaks $12^{\prime}$ and $15^{\prime}$.

\section{Materials and Methods}

\subsection{Materials and Methods for Phytochemistry Research}

\subsubsection{General Experimental Procedures}

UV and IR spectra were recorded on a Varian Cary 50 UV-Vis and Varian 640-IR FT-IR spectrophotometer, respectively. Optical rotations were measured on a Rudolph Autopol ${ }^{\circledR}$ IV automatic polarimeter. NMR spectra were determined on a Bruker $500 \mathrm{MHz}$ NMR spectrometer at $500 \mathrm{MHz}$ for ${ }^{1} \mathrm{H}$ and $125 \mathrm{MHz}$ for ${ }^{13} \mathrm{C}-\mathrm{NMR}$ (internal standard: TMS). Negative-ion mode ESI-Q-Orbitrap MS were obtained on a Thermo UltiMate 3000 UHPLC instrument (Thermo, Waltham, MA, USA).

Column chromatographies (CC) were performed on macroporous resin D101 (Haiguang Chemical Co., Ltd., Tianjin, China), silica gel (48-75 $\mu \mathrm{m}$, Qingdao Haiyang Chemical Co., Ltd., Qingdao, China), ODS (40-63 m, YMC Co., Ltd., Tokyo, Japan), and Sephadex LH-20 (Ge Healthcare Bio-Sciences, Uppsala, Sweden). Preparative high performance liquid chromatography (pHPLC) column, Cosmosil 5C 18 -MS-II (20 mm i.d. $\times 250 \mathrm{~mm}$, Nakalai Tesque, Inc., Tokyo, Japan) were used to separate the constituents.

\subsubsection{Plant Material}

The fresh aerial parts of Allium mongolicum Regel were collected from Alxa League, Inner Mongolia Autonomous Region, China, and identified by Dr. Li Tianxiang (The Hall of TCM Specimens, Tianjin 
University of TCM, China). The voucher specimen was deposited at the Academy of Traditional Chinese Medicine of Tianjin University of TCM.

\subsubsection{Extraction and Isolation}

See supporting information.

\subsubsection{Acid Hydrolysis of 1, 3, 4 and 6}

Solution of 1, 3, 4 and 6 (each $2.0 \mathrm{mg}$ ) in $5 \%$ aqueous $\mathrm{H}_{2} \mathrm{SO}_{4}$-1,4-dioxane were heated under reflux for $1 \mathrm{~h}$, respectively. After cooling, the reaction mixture was neutralized with Amberlite IRA-400 $\left(\mathrm{OH}^{-}\right.$form), removed by filtration, subjected to ODS CC $\left(\mathrm{H}_{2} \mathrm{O}\right)$, and the $\mathrm{H}_{2} \mathrm{O}$ eluate was reacted with L-cysteine methyl ester hydrochloride in pyridine and $\mathrm{N}, \mathrm{O}$-bis(trimethylsilyl)trifluoroacetamide (BSTFA), successively. Finally, the reaction product was elucidated by GC analysis (GC conditions, column: Agilent Technologies INC Catalog 19,091 J-413 HP-5, $30 \mathrm{~m} \times 0.320 \mathrm{~mm}$ (i.d.) capillary column; column temperature: $230{ }^{\circ} \mathrm{C}$; carrier gas: $\mathrm{N}_{2}$ ), and D-glucuronic acid and D-glucose hydrolysates were identified from 1, 3, and 6; D-glucuronic acid, D-glucose, as well as L-rhamnose hydrolysates were detected from 4 by comparing it retention times $\left(t_{\mathrm{R}}\right.$ : D-glucuronic acid, $23.3 \mathrm{~min}$; D-glucose, $19.6 \mathrm{~min}$; L-rhamnose, $11.4 \mathrm{~min}$ ) with those of their authentic samples treated in the same way.

\subsubsection{Acid Hydrolysis of $\mathbf{2}, \mathbf{5}$ and $\mathbf{7 - 1 0}$}

The solution of compounds $\mathbf{2}, \mathbf{5}$ and $\mathbf{7 - 1 0}$ (each $1.5 \mathrm{mg}$ ) in $1 \mathrm{M} \mathrm{HCl}(1.0 \mathrm{~mL}$ ) was heated under reflux for $3 \mathrm{~h}$. After cooling, the reaction mixture was neutralized with Amberlite IRA-400 ( $\mathrm{OH}^{-}$form), then analyzed by HLPC [column, Kaseisorb $\mathrm{LC} \mathrm{NH}_{2}-60-5,4.6 \mathrm{~mm}$ i.d. $\times 250 \mathrm{~mm}$ (Tokyo Kasei Co., Ltd., Tokyo, Japan); mobile phase, $\mathrm{CH}_{3} \mathrm{CN}-\mathrm{H}_{2} \mathrm{O}(75: 25, v / v$; flow rate, $\left.1.0 \mathrm{~mL} / \mathrm{min})\right]$. As a result, D-glucose was detected from the aqueous phase of $\mathbf{2 , 5}$ and $\mathbf{7 - 1 0}$ by comparison of its retention time and optical rotation with those of the authentic sample, D-glucose $\left(t_{\mathrm{R}} 12.5 \mathrm{~min}\right.$, positive), respectively.

\subsection{Materials and Methods for Bioassay}

The activities of compounds 1-31 were tested for frequency and height by using tissue perfusion method reported before [30]. Samples in DMSO solution were added after 15 min equilibrate incubation; the final DMSO concentration was $0.1 \%$ and final concentration of samples were $50 \mu \mathrm{M}$. Mosapride citrate dihydrate (Xi'an Janssen Pharmaceutical Ltd., Xi'an, China), final concentration was $200 \mu \mathrm{g} / \mathrm{mL}$.

Data were analyzed by SPSS 22.0 software. All values were expressed as mean \pm S.D. A $p$-value of 0.05 was considered to indicate statistical significance. One-way analysis of variance (ANOVA) and Tukey's studentized range test were used for the evaluation of the significant differences between means and post hoc, respectively.

\subsection{Materials and Methods for Qualitative Analysis}

\subsubsection{Materials}

The isolated 31 compounds including 24 flavonoids, and 7 phenolic acids were used for reference standards. Their purities were $>98 \%$.

HPLC grade Acetonitrile (Thermo-fisher, Waltham, MA, USA), formic acid (Roe Scientific Inc., Newark, NJ, USA), and ultra-pure water prepared with a Milli-Q purification system (Millipore, MA, USA) were used for LC-MS analysis. 


\subsubsection{Sample Preparation}

\section{Preparation of Standard Solutions}

Standard test solutions of the above-mentioned standard references were prepared in $\mathrm{MeOH}$ at a final concentration of $1 \mu \mathrm{g} / \mathrm{mL}$ approximately. All stock solutions were stored at $4{ }^{\circ} \mathrm{C}$ in darkness and brought to room temperature before use.

Preparation of the Aerial Parts of A. mongolicum Extract Test Solutions

A. mongolicum extract (AM) was prepared by using the same method as described in "Extraction and Isolation" section. The AM was dissolved with $\mathrm{MeOH}$ and filtered with $0.22 \mu \mathrm{m}$ microporous membrane to get test stock solution at a final concentration of $30 \mathrm{mg} / \mathrm{mL}$, which was stored at $4{ }^{\circ} \mathrm{C}$ in darkness and brought to room temperature before use.

\subsubsection{UHPLC}

A Thermo UltiMate 3000 UHPLC instrument (Thermo, Waltham, MA) equipped with a quaternary pump, an autosampler was used to accomplish the analysis. Samples were separated on a Waters ACQUITY UPLC®HSS C18 $(2.1 \times 100 \mathrm{~mm}, 1.8 \mu \mathrm{m})$ using a mobile phase composed of $\mathrm{H}_{2} \mathrm{O}$ with $0.1 \%$ formic acid (A) and $\mathrm{CH}_{3} \mathrm{CN}$ with $0.1 \%$ formic acid (B) in the gradient program: 0-2 min, 9-10\% $\mathrm{B}$; 2-5 min, 10-17\% B; 5-7 min, 17-20\% B; 7-9 min, 20\% B; 9-10 min, 20-86\% B; 10-14 min, 86-100\% B; 14-17 $\mathrm{min}, 100 \% \mathrm{~B}$; An equilibration of $3 \mathrm{~min}$ was used between successive injections. The flow rate was $0.4 \mathrm{~mL} / \mathrm{min}$, and column temperature was $35^{\circ} \mathrm{C}$. An aliquot of $1 \mu \mathrm{L}$ of each sample was injected for analysis.

\subsubsection{ESI-Q-Orbitrap MS and Automatic Components Extraction}

For tandem mass spectrometry analysis, a Thermo ESI-Q-Orbitrap MS mass spectrometer was connected to the UltiMate 3000 UHPLC instrument via ESI interface. Ultra-high purity nitrogen $\left(\mathrm{N}_{2}\right)$ was used as the collision gas and the sheath/auxiliary gas. The ESI source parameters were set as follows: ion spray voltage $3.2 \mathrm{kV}$, capillary temperature $350{ }^{\circ} \mathrm{C}$, ion source heater temperature $300{ }^{\circ} \mathrm{C}$, sheath gas $\left(\mathrm{N}_{2}\right) 40 \mathrm{~L} / \mathrm{h}$, auxiliary gas $\left(\mathrm{N}_{2}\right) 10 \mathrm{~L} / \mathrm{min}$, and a normalized collision energy (NCE) of $-35 \mathrm{~V}$ was used. The Orbitrap analyzer scanned the mass range from $\mathrm{m} / \mathrm{z} 150$ to 1500 in negative ion mode. Monitoring time was 0-17 min. Detection was obtained by full mass-dd mass mode. The MS data were recorded in both profile and centroid formats. Data recording and processing were performed using the Xcalibur 4.0 software (Thermo Fisher Scientific, Inc., Waltham, MA, USA). The accuracy error threshold was fixed at 5 ppm.

Software-aided, automatic background subtraction and components extraction technique was used to generate a peak list containing all the components profiled from the aerial part of A. mongolicum. Sieve v2.2 SP2 (Thermo Fisher Scientific) was used for the automatic components extraction: time range, 1-17 min; BP minimum count, 10,000; BP minimum scans, 5; Background SN, 3; MZ Step, 10; and Frame, $>1$.

\section{Conclusions}

This paper displayed a study - the first of its kind-focused on the systematic bioactive constituents of the aerial parts of $A$. mongolicum in the gastrointestinal tract. As a result, AM and AMH showed a significant increase in the contraction amplitude of mouse small intestinal muscles, which indicated they might have therapeutic effects on constipation. During this process, we made several achievements:

The first comprehensive phytochemistry investigation was developed for AM by using various spectral and chromatographic methods: six new flavonoid glycosides, mongoflavonosides $A_{1}(1)$, $A_{2}(2), A_{3}(3), A_{4}(4), B_{1}(5), B_{2}(6)$, four new phenolic acid glycosides, mongophenosides $A_{1}(7), A_{2}$ 
(8), $A_{3}(9), B(10)$, as well as 21 known compounds were yielded. They were mainly flavonoids and phenolic acids.

The flavonoids and phenolic acids were clarified for the first time to be the main bioactive constituents of $A$. mongolicum on gastrointestinal tract: flavonoids 3, 4, 11-15, 21-23, and 26, as well as phenolic acids 7, 29, and 30 showed significant increase in the height of mouse small intestinal muscle.

Furthermore, a fast analysis method for flavonoids and phenolic acids in the aerial parts of A. mongolicum was established for the first time by using LC-MS. According to $t_{R}$ and $m / z, 31$ compounds (1-31) were unambiguously identified by comparing to the standard references. Then, on the basis of generalized rules of MS/MS fragmentation pattern, chromatographic behaviors, as well as their biosynthetic laws, five flavonoid glycosides (32-36) and one phenolic acid glycoside (37) were tentatively speculated. Among them, peak $\mathbf{3 6}$ was a potential new one. Thus, the first evidence for quality control of A. mongolicum has been duly provided.

Supplementary Materials: Supplementary data (The NMR and HRESIMS spectra of compounds 1-10, as well as extraction and isolation process) associated with this article can be found in the online version.

Author Contributions: Y.Z. (Yi Zhang) and T.W. designed the research and wrote the manuscript; Y.D., J.R., and Z.D. performed the experimental work; W.Z. and H.J. corrected the data and reviewed the literatures; M.H. and Y.Z. (Ying Zhang) perfected the language. All authors discussed, edited, and approved the final version. All authors have read and agreed to the published version of the manuscript.

Funding: This research was supported by Program for National Natural Science Foundation of China (81673688), and the Important Drug Development Fund, Ministry of Science and Technology of China (2018ZX09711001-009-010, 2018ZX09735-002).

Conflicts of Interest: The authors declare no conflict of interest.

\section{References}

1. Gulzar, A.; Siddiqui, M.B.; Bi, S. Phenolic acid allelochemicals induced morphological, ultrastructural, and cytological modification on Cassia sophera L. and Allium cepa L. Protoplasma 2015, 253, 1211-1221. [CrossRef] [PubMed]

2. Chang, T.-C.; Jang, H.-D.; Lin, W.-D.; Duan, P.-F. Antioxidant and antimicrobial activities of commercial rice wine extracts of Taiwanese Allium fistulosum. Food Chem. 2016, 190, 724-729. [CrossRef] [PubMed]

3. Onyeoziri, U.P.; Romanus, E.N.; Onyekachukwu, U.I. Assessment of antioxidant capacities and phenolic contents of nigerian cultivars of onions (Allium cepa L) and garlic (Allium sativum L). Pak. J. Pharm. Sci. 2016, 29, 1183-1188. [PubMed]

4. Bondonno, N.P.; Dalgaard, F.; Kyrø, C.; Murray, K.; Bondonno, C.P.; Lewis, J.R.; Croft, K.D.; Gislason, G.; Scalbert, A.; Cassidy, A.; et al. Flavonoid intake is associated with lower mortality in the Danish Diet Cancer and Health Cohort. Nat. Commun. 2019, 10, 3651-3661. [CrossRef]

5. Ivanovna, L.; Mikhailovna, T.; Vladimirovich, D.; Valentinovich, M.; Aleksandrovna, E.; Babaskina, L.I.; Litvinova, T.M.; Babaskin, D.V.; Kiselevsky, M.V.; Savinova, O.V.; et al. Influence of Flavonoids on the Cytotoxic Activity of Mononuclear Blood Cells in Model Tests. Open Access Maced. J. Med. Sci. 2019, 7, 1900-1904. [CrossRef]

6. Li, Y.-D.; Guan, J.-P.; Tang, R.-C.; Qiao, Y.-F. Application of Natural Flavonoids to Impart Antioxidant and Antibacterial Activities to Polyamide Fiber for Health Care Applications. Antioxidants 2019, 8, 301. [CrossRef]

7. Wang, W.; Li, J.; Zhang, H.; Wang, X.; Fan, J.; Zhang, X. Phenolic compounds and bioactivity evaluation of aqueous and methanol extracts of Allium mongolicum Regel. Food Sci. Nutr. 2019, 7, 779-787. [CrossRef]

8. Chen, Y.-A.; Tsai, J.-C.; Cheng, K.-C.; Liu, K.-F.; Chang, C.-K.; Hsieh, C.-W. Extracts of black garlic exhibits gastrointestinal motility effect. Food Res. Int. 2018, 107, 102-109. [CrossRef]

9. Zhang, Y.; Huang, P.; He, W.; Sakah, K.J.; Ruan, J.; Li, Z.; Wang, T. Bioactive constituents obtained from the fruits of Citrus aurantium. J. Nat. Med. 2019, 73, 146-153.

10. He, W.; Liu, M.; Li, Y.; Yu, H.; Wang, D.; Chen, Q.; Chen, Y.; Zhang, Y.; Wang, T. Flavonoids from Citrus aurantium ameliorate TNBS-induced ulcerative colitis through protecting colonic mucus layer integrity. Eur. J. Pharmacol. 2019, 857, 172456. [CrossRef] 
11. He, W.; Li, Y.; Liu, M.; Yu, H.; Chen, Q.; Chen, Y.; Ruan, J.; Ding, Z.; Zhang, Y.; Wang, T. Citrus aurantium L. and Its Flavonoids Regulate TNBS-Induced Inflammatory Bowel Disease through Anti-Inflammation and Suppressing Isolated Jejunum Contraction. Int. J. Mol. Sci. 2018, 19, 3057. [CrossRef] [PubMed]

12. Qi, S.; Wang, T.; Chen, R.; Wang, C.; Ao, C. Effects of flavonoids from Allium mongolicum Regel on growth performance and growth-related hormones in meat sheep. Anim. Nutr. 2017, 3, 33-38. [CrossRef] [PubMed]

13. Du, H.; Erdene, K.; Chen, S.; Qi, S.; Bao, Z.; Zhao, Y.; Wang, C.; Zhao, G.; Ao, C.; Khas, E.; et al. Correlation of the rumen fluid microbiome and the average daily gain with a dietary supplementation of Allium mongolicum Regel extracts in sheep1. J. Anim. Sci. 2019, 97, 2865-2877. [CrossRef]

14. Li, M.-Y.; Guo, W.-Q.; Guo, G.-L.; Zhu, X.-M.; Niu, X.-T.; Shan, X.-F.; Tian, J.-X.; Wang, G.-Q.; Zhang, D.-M. Effect of sub-chronic exposure to selenium and Allium mongolicum Regel flavonoids on Channa argus: Bioaccumulation, oxidative stress, immune responses and immune-related signaling molecules. Fish Shellfish. Immunol. 2019, 91, 122-129. [CrossRef]

15. Wang, T.; Wang, C.; Dan, N.; Sa, R.; Du, H.; Guo, C.; Khas-Erdene; Cao, Q.; Ao, C. Effects of total flavonoids from Allium mongolicum Regel on inflammatory mediators induced by lipopolysaccharide of mouse peritoneal macrophages. Dongwu Yingyang Xuebao 2018, 30, 3702-3709.

16. Zhao, C. Studies on Separation, Purification and Structural Characterizations of Flavonoids from Allium mongolium Regel and Its Effects on Immunity and Antioxidant Function in Mice; Inn. Mongolia Agricultural University: Hohhot, China, 2008.

17. Dong, Y.; Qu, L.; Li, X.; Han, L.; Wang, T.; Zhang, Y. Isolation and identification of constituents from Allium mongolicum Regel I. Zhongguo Yaowu Huaxue Zazhi 2015, 25, 298-302.

18. Dong, Y.; Shi, W.; Yang, S.; Li, X.; Zhang, Y.; Wang, T. Isolation and structural identification of constituents from Allium mongolicum Regel II. Tianjin Zhongyiyao Daxue Xuebao 2016, 35, 404-408.

19. Manguro, L.O.A.; Ugi, I.; Lemmen, P.; Hermann, R. Flavonol glycosides of Warburgia ugandensis leaves. Phytochem. 2003, 64, 891-896. [CrossRef]

20. Budzianowski, J. Six flavonol glucuronides from Tulipa gesneriana. Phytochemistry 1991, 30, 1679-1682. [CrossRef]

21. Kokubo, T.; Nakamura, M.; Yamakawa, T.; Noguchi, H.; Kodama, T. Quercetin 3,7,4'-triglucoside formation from quercetin by Vitis hybrid cell cultures. Phytochemistry 1991, 30, 829-831. [CrossRef]

22. Yi, Y.; Wu, X.; Wang, Y.; Ye, W.-C.; Zhang, Q.-W. [Studies on the flavonoids from the flowers of Hylocereus undatus]. Zhong Yao Cai = Zhongyaocai = J. Chin. Med. Mater. 2011, 34, 712-716.

23. Hardorne, J.B. Plant polyphenols-XI. The structure of acylated anthocyanins. Phytochemistry 1964, 3, 151-160. [CrossRef]

24. Han, S.H.; Suh, W.S.; Park, K.J.; Kim, K.H.; Lee, K.R. Two new phenylpropane glycosides from Allium tuberosum Rottler. Arch. Pharmacal. Res. 2015, 38, 1312-1316. [CrossRef] [PubMed]

25. Fei, Y.; Chen, Z.; Li, X.; Xu, Q.; Yang, S. Chemical constituents from seeds of Helianthus annuus. Zhongcaoyao 2014, 45, 631-634.

26. Chan, J.; Yang, L.; Rena, K. Studies on chemical constituents from EtOAc fraction of Sorbus tiansschanica. Zhongguo Zhongyao Zazhi 2009, 34, 175-176.

27. Zhang, Y.; Li, X.; Ruan, J.; Wang, T.; Dong, Y.; Hao, J.; Liu, E.; Han, L.; Gao, X.; Wang, T. Oleanane type saponins from the stems of Astragalus membranaceus (Fisch.) Bge. var. mongholicus (Bge.) Hsiao. Fitoterapia 2016, 109, 99-105. [CrossRef]

28. Yang, S.; Sun, F.; Ruan, J.; Yan, J.; Huang, P.; Wang, J.; Han, L.; Zhang, Y.; Wang, T. Anti-inflammatory constituents from Cortex Dictamni. Fitoterapia 2019, 134, 465-473. [CrossRef]

29. Lee, K.R.; Jeong, E.-K.; Choi, S.U.; Hong, J.; Lee, I.K. New Flavonol Glycosides from Cardamine komarovii. Heterocycles 2011, 83, 2615. [CrossRef] 
30. Shi, W.; Ruan, J.; Guo, Y.; Ding, Z.; Yan, J.; Qu, L.; Zheng, C.; Zhang, Y.; Wang, T. Bioactive constituents study of Pugionium cornutum L. Gaertn on intestinal motility. Fitoterapia 2019, 138, 104291. [CrossRef]

31. Ruan, J.; Yan, J.; Zheng, D.; Sun, F.; Wang, J.; Han, L.; Zhang, Y.; Wang, T. Comprehensive Chemical Profiling in the Ethanol Extract of Pluchea indica Aerial Parts by Liquid Chromatography/Mass Spectrometry Analysis of Its Silica Gel Column Chromatography Fractions. Molecules 2019, 24, 2784. [CrossRef]

Sample Availability: Samples of all compounds are available from the authors.

(C) 2020 by the authors. Licensee MDPI, Basel, Switzerland. This article is an open access article distributed under the terms and conditions of the Creative Commons Attribution (CC BY) license (http://creativecommons.org/licenses/by/4.0/). 